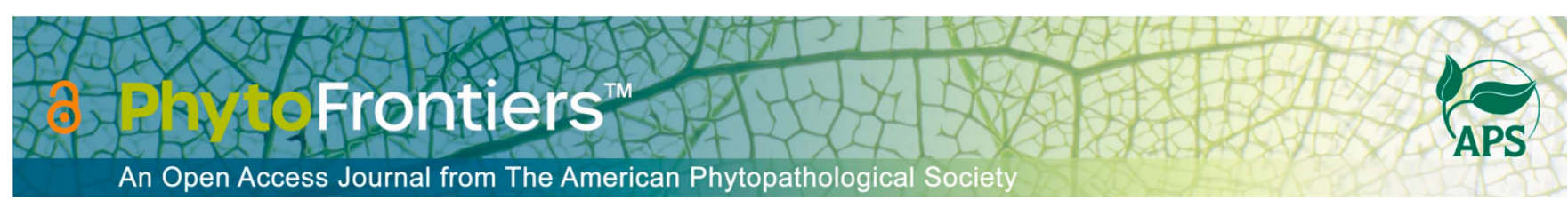

\title{
Research
}

\section{Verticillium klebahnii and V. isaacii Isolates Exhibit Host-Dependent Biological Control of Verticillium Wilt Caused by V. dahliae}

\author{
Krishna D. Puri ${ }^{1}$ (1) | Xiaoping $\mathrm{Hu}^{2}$ (1) | Suraj Gurung ${ }^{1}$ (1) | Dylan P. G. Short ${ }^{1}$ | German V. Sandoya ${ }^{3}$ (i) | \\ Marion Schild ${ }^{4} \mid$ Yuanyuan Zhang ${ }^{5}$ | Jun Zhao ${ }^{5}$ | Amy G. Anchieta ${ }^{6}$ | Steven J. Klosterman ${ }^{6}$ (D) | \\ Krishna V. Subbarao ${ }^{1,+}$ (i)
}

\footnotetext{
1 Department of Plant Pathology, University of California, Davis, 1636 E. Alisal St., Salinas, CA 93905, U.S.A.

${ }^{2}$ State Key Laboratory of Crop Stress Biology for Arid Areas and College of Plant Protection, Northwest A\&F University, Yangling 712100, China

${ }^{3}$ Horticultural Science Department, Everglades Research and Education Center, University of Florida - Institute of Food and Agriculture Sciences, Belle Glade, FL, 33430, U.S.A.

${ }^{4}$ Swiss Federal Institute of Technology Zurich, Ramistrasse 101, Zurich 8092, Switzerland

${ }^{5}$ College of Horticulture and Plant Protection, Inner Mongolia Agricultural University, Hohhot, 010018 , China

${ }^{6}$ United States Department of Agriculture-Agricultural Research Service, Crop Improvement and Protection Research Unit, Salinas, CA, U.S.A
}

\footnotetext{
${ }^{\dagger}$ Corresponding author: K. V. Subbarao kvsubbarao@ucdavis.edu
}

Accepted for publication 1 April 2021.

\section{Funding}

Support for this research work was provided by the California Leafy Greens Research Board and, in part, by the United States Department of Agriculture-National Institute for Food and Agriculture (USDA-NIFA) Specialty Crops Research Initiative grant number 2010-51181-21069 and USDA-NIFA grant number 59-5305-4-002.

K. D. Puri and X. Hu contributed equally to this study.

The author(s) declare no conflict of interest.

\begin{abstract}
Verticillium dahliae, the soilborne fungal pathogen, causes vascular wilt on many economically important crops, resulting in significant yield losses. V. klebahnii (isolate PD659) and V. isaacii (isolate PD660), two related species that cause few or no symptoms in some hosts, were evaluated as potential biocontrol agents (BCAs) in eggplant, lettuce, and tomato by pre-, post-, and coinoculation with a virulent race 1 isolate of $V$. dahliae (VdLs16). Initial studies demonstrated that the biocontrol efficacy of both BCAs was similar to reference BCA Talaromyces flavus (NRRL15936) across all hosts $(\alpha=0.05)$. Subsequent experiments with PD659 against $V$. dahliae isolate Sm113 from eggplant, VdLs16 and VdLs17 isolates from lettuce, and Le1811 isolate from tomato demonstrated a significant biocontrol efficacy in eggplant and tomato but not in lettuce (at 95\% confidence interval), suggesting host-dependent effectiveness of $V$. klebahnii. Confocal microscopy using green fluorescent protein-tagged tomato $V$. dahliae isolate Le1811 indicated delayed xylem colonization or lack of pathogen progression into the vascular system in a host-dependent manner on BCA-treated plants. Quantitative analyses of the expression of defense-related genes PR1a, PR5, acidic extracellular $\beta$-1,3-glucanase (G/ucA), basic intracellular $\beta$-1,3-glucanase (GlucB), acidic extracellular chitinase (Chi3), basic intracellular chitinase (Chi9), and cysteine proteases (cysProteases) in tomato in the presence or absence of PD659 suggested an elevated expression of defense-related genes in compatible interaction of $V$. dahliae-tomato cultivar Early Pak. V. klebahnii (PD659) may delay the entry of $V$. dahliae by competing for space or nutrients during the initial stages of root colonization.
\end{abstract}

Keywords: biocontrol, disease management, vegetable crops, Verticillium wilt, Verticillium klebahnii, V. isaacii 
Verticillium dahliae is a destructive soilborne fungal pathogen that causes vascular wilt in more than 400 plant species, including lettuce, strawberry, tomato, eggplant, and other economically important crops (Atallah et al. 2011; Berlanger and Powelson 2000; Pegg and Brady 2002; Subbarao et al. 2017). Despite the broad host range, many isolates display differential virulence on some of these hosts (Gurung et al. 2015). The pathogen produces melanized microsclerotia, which serve as resting structures and remain viable in the soil for over 10 years to initiate disease in the subsequent crops (Klosterman et al. 2009; Vallad and Subbarao 2008). Losses caused by $V$. dahliae exceed several billion dollars worldwide annually (Klosterman et al. 2009; Pegg and Brady 2002).

Historically, the management of soilborne pathogens, including $V$. dahliae, has relied on crop rotation, resistant cultivars, and soil fumigation. However, the unavailability of resistant cultivars, prohibition of methyl bromide (MB) use for agricultural soil fumigation, ineffective cultural practices, broad host range, and prolonged survival of the dormant propagule have all impeded the successful management of this disease (Hayes et al. 2011a; Usami et al. 2017). Soil fumigation using MB was the primary method of controlling Verticillium wilt before its complete phase-out from agricultural fumigation in 2015 owing to its ozone-depleting properties and high toxicity to humans, animals, and the environment (Chellemi et al. 2016; Martin 2003). Currently, a few chemical alternatives to $\mathrm{MB}$ are available but are not as effective (Duniway 2002; Meadows 2013). Increased demand for organic produce or crops and environmental hazards associated with chemicals has reduced their use in agriculture (Donley 2019).

Biological pest management using natural antagonists and niche competitors has garnered considerable research attention for sustainable crop production (Bale et al. 2008). Biological antagonism works against pathogens through various direct mechanisms such as antibiosis and competition for rhizosphere space and infection sites or nutrients, or indirectly by inducing plant growth and systemic disease resistance (Deketelaere et al. 2017; Fravel 2005; Li et al. 2013; Malandraki et al. 2008; Veloso and Díaz 2012). Even a single biocontrol agent (BCA) functions through several complementary mechanisms (Deketelaere et al. 2017). An induced host defense response triggered by BCAs is one of the established mechanisms for suppressing plant pathogens. Host defense activation via pathogen-associated molecular patterns in cotton (Han et al. 2015), defenserelated pathogenesis-related $(P R)$ genes $P R 1$ and $P R 4$ in eggplant (Angelopoulou et al. 2014), induced plant resistance through salicylic and jasmonate-dependent defense pathways (Gkizi et al. 2016; Tjamos et al. 2005), and damage-associated pattern-triggered production of endopolymethylgalacturonase, endoglucanase, endoxyloglucanase (Gkizi et al. 2016), and so on are proven defense mechanisms against $V$. dahliae.

Fungal antagonists such as Gibellulopsis nigrescens (Zare et al. 2007), Fusarium oxysporum, and Talaromyces flavus (anamorph Penicillium dangeardii) have shown efficacy against Verticillium wilt in many crops but none is currently employed in commercial agriculture (Angelopoulou et al. 2014; Marois et al. 1984; Veloso and Díaz 2012; Zhu et al. 2013). Application of Paenibacillus alvei K165 and nonpathogenic $F$. oxysporum F2 reduced Verticillium wilt in eggplant (Angelopoulou et al. 2014) by limiting the germination of microsclerotia in the rhizosphere (Antonopoulos et al. 2008). Microsclerotial germination of $V$. dahliae was also lowered by Gliocladium roseum and T. flavus strains (Fravel 1989; Keinath et al. 1991; Kim et al. 1988). Similarly, the nonpathogenic isolate F. oxysporum 47 (Fo47) conferred protection against $V$. dahliae and is associated with the expression of defense genes (Veloso and Díaz 2012). Gibellulopsis nigrescens significantly reduced the Verticillium wilt symptoms in cotton (Zhu et al. 2013). Among several characterized fungal antagonists, T. flavus is one of the more broadly used and reliable BCAs to manage Verticillium wilt on eggplant, potato, cotton, and tomato (Li et al. 2013; Marois et al. 1982, 1984; Naraghi et al. 2010; Tjamos and Fravel 1995).

Specific isolates or species within the genus Verticillium can colonize their hosts with no or minimal vascular discoloration without the external wilt-like symptoms (Chen et al. 2004; Malcolm et al. 2013; Qin et al. 2008; Robinson et al. 2007). $V$. tricorpus suppressed Verticillium wilt of potato and cauliflower (Davis et al. 2000; França et al. 2013). Despite $V$. tricorpus being endophytic or weakly pathogenic in potato and offering protection against $V$. dahliae (Davis and Sorensen 1985; Davis et al. 2000), some isolates were highly pathogenic on lettuce (Usami et al. 2011). Using genealogical concordance phylogenetic species recognition (Taylor et al. 2000), three different lineages were recognized within the morphospecies $V$. tricorpus: V. klebahnii, V. isaacii, and V. tricorpus sensu stricto (Inderbitzin et al. 2011). $V$. isaacii Vt305 was effective in reducing Verticillium wilt of cauliflower caused by $V$. longisporum in both the greenhouse and the field (Deketelaere et al. 2020). Therefore, it is quite likely that some of the previously reported weakly pathogenic or nonpathogenic isolates of $V$. tricorpus belong to either $V$. isaacii or $V$. klebahnii. In cauliflower, the endophytic $V$. isaacii isolate $\mathrm{Vt} 305$ colonized the roots, hypocotyl, and stem without visible wilting and significantly reduced wilt severity caused by $V$. longisporum (Tyvaert et al. 2014).

Previously, we had isolated the endophytic V. isaacii (PD660) and $V$. klebahnii (PD659) isolates from the roots of spinach and lettuce, respectively (Short et al. 2015). Both V. klebahnii PD659 (Gurung et al. 2015) and $V$. isaacii (PD660) (unpublished) produced no visible symptoms on lettuce and tomato, and inoculated plants seldom exhibited vascular discoloration. Thus, it is essential to explore their endophytic lifestyles in the roots and stems of lettuce, tomato, or other hosts and to test their biocontrol efficacy against pathogenic Verticillium spp. In the current study, we tested the biocontrol potential of nonpathogenic $V$. isaacii (PD660) and $V$. klebahnii (PD659) isolates on eggplant, lettuce, and tomato, initially challenging them against a single $V$. dahliae isolate (VdLs16) as pre-, post-, and coinoculated treatments and compared their efficacy with a known BCA, T. flavus NRRL15936. Subsequently, the efficacy of isolates PD659 and PD660 was further tested against a panel of $V$. dahliae isolates derived from three different hosts. The specific objectives of the study were to (i) evaluate the biocontrol efficacy of $V$. isaacii (PD660) and $V$. klebahnii isolate (PD659) in reducing $V$. dahliae colonization and wilt in eggplant, lettuce, and tomato; (ii) explore biocontrol mechanisms based on confocal microscopy using green fluorescent protein (GFP)-tagged isolates of $V$. dahliae during the infection process; and (iii) determine whether $V$. klebahnii isolate PD659 induces the plant defense-related genes during the infection process in tomato and explore the possible mode of action.

\section{MATERIALS AND METHODS}

\section{Fungal isolates and maintenance}

Pathogenic V. dahliae isolates VdLs16 (race 1) and VdLs17 (race 2) from lettuce, Sm113 (race 2) from eggplant, and Le1811 (race 2) from tomato were isolated from symptomatic 
plants collected form commercial farms in California, United States. The biocontrol isolates PD659 (V. klebahnii) and PD660 ( $V$. isaacii) were isolated from lettuce roots and spinach plants sampled at Salinas, CA, respectively, as described previously by Short et al. (2015). The reference BCA T. flavus strain NRRL15936 was obtained from the United States Department of Agriculture Culture Collection (Peoria, IL, U.S.A.). All fungal isolates were grown on potato dextrose agar (PDA) (Difco Laboratories Inc., Detroit, MI, U.S.A.) and used for inoculation or storage in $25 \%$ glycerol at $-80^{\circ} \mathrm{C}$.

\section{Biocontrol experiments using $V$. dahliae isolate VdLs16 from lettuce}

Seed of eggplant cultivar Long Purple, tomato cultivar Early Pak, and lettuce cultivar Salinas were planted in 50-well trays filled with Sunshine Professional Growing Mix 4 (SUNGRO Horticulture, Canada). In all hosts, 2-week-old seedlings were inoculated with $3 \mathrm{ml}$ of a suspension of $1.0 \times 10^{7}$ conidia/ml in the germination tray, then transplanted into 0.5 liter Styrofoam cups (Amerifoods, USA) filled with steamsterilized sand/potting soil $(2: 1[\mathrm{vol} / \mathrm{vol}])$ mixture.

In the first experiment, five treatment combinations each for the three putative BCAs plus five control treatments (for a total of 20 treatments) were used to evaluate the biocontrol efficacy against $V$. dahliae isolate VdLs16 relative to the control BCA T. flavus strain NRRL15936 (Fig. 1) in three different hosts. Treatments included were (i) inoculation of VdLs16 1 week prior to BCAs, (ii) inoculation of VdLs16 2 weeks prior to BCAs, (iii) coinoculation of BCAs and VdLs16, (iv) inoculation of BCAs 1 week prior to VdLs16, and (v) inoculation of BCAs 2 weeks prior to VdLs16. Seedlings treated with sterile distilled water served as negative controls, while plants inoculated only with VdLs16, PD659, PD660, and strain NRRL15936 served as positive controls. Following seedling transplantation, individual cups were arranged in a randomized complete block design in factorial arrangement using three replications, each with at least seven plants (21 total plants). The entire experiment was repeated once. Seeding, inoculation, disease rating, and standard agronomic practices were performed the same way across experiments (Puri et al. 2017).

\section{Biocontrol experiments using host-dependent isolates}

The second experiment was conducted to test the efficacy of both BCAs (PD659 and PD660) using the treatment that reduced Verticillium wilt the most in the experiment above. Each host was inoculated with isolates obtained form infected root or stem tissues of the corresponding host: eggplant isolate Sm113 on Long Purple eggplant, lettuce isolates VdLs16 and VdLs17 on Salinas lettuce, and tomato isolate Le1811 on Early Pak tomato. In this experiment, BCAs were introduced on 2-week-old seedlings and later challenged by individual

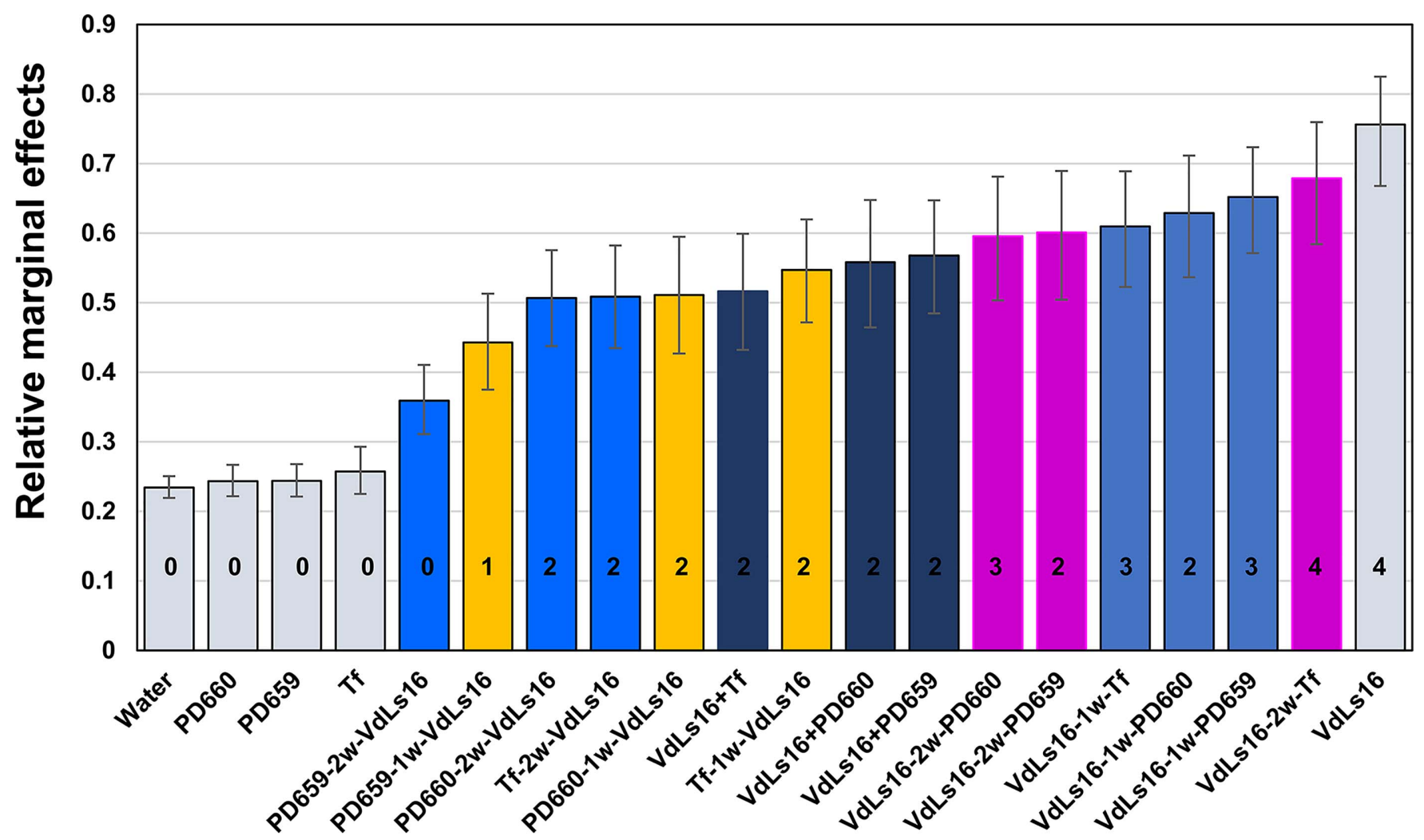

FIGURE 1

Relative marginal effect (RME) and median disease severity scores combined across eggplant, lettuce, and tomato. Nonoverlapping confidence interval $(\mathrm{Cl})$ lines on the top of the bars represent statistically significant treatment effects at $95 \% \mathrm{Cl}$. Numbers inside the bars represent median disease severity, while the color of bars represents timing of treatments. The treatment annotations represent the order and the interval in which inoculations of the hosts were performed. For example, PD659-1w-VdLs16 represents PD659 applied 1 week before VdLs16, and VdLs16-2w-PD659 represents VdLs16 applied 2 weeks before PD659, and so on. The plus (+) sign represents coinoculation at the same time. $T f=$ Talaromyces flavus NRRL15936. Both PD659 (Verticillium klebahnii) and VdLs16 (V. dahliae) were obtained from lettuce, while isolate PD660 (V. isaacii) was collected from spinach. 
pathogenic isolates at 2 weeks after $\mathrm{BCA}$ treatments. The BCAs were introduced by immersing the seedling roots in a suspension of $1.0 \times 10^{7}$ conidia $/ \mathrm{ml}$ for $10 \mathrm{~min}$; then, seedlings were transplanted into Styrofoam cups as described above. The pathogenic isolates were inoculated by drenching the soil with a $5-\mathrm{ml}$ inoculum suspension containing $1.0 \times 10^{7}$ conidia $/ \mathrm{ml} /$ cup. Seedlings that received water treatment at the time of BCA inoculation (2-week-old seedlings) but were challenged with the pathogenic isolate 2 weeks later (4-week-old seedlings) served as positive controls. The water-treated plants served as negative controls. The experimental design was a randomized complete block design with three replications each as above, and the experiment was repeated once.

\section{GFP transformants}

$V$. dahliae isolate Le1811 (race 2) from tomato was transformed with a GFP gene using the Agrobacterium tumefaciensmediated transformation (ATMT) protocol (Maruthachalam et al. 2013). Briefly, conidia from a 7-day-old culture were harvested, diluted to approximately $1.0 \times 10^{6}$ conidia $/ \mathrm{ml}$, and used for ATMT. The transformation was carried out using a T-DNA binary vector (pSK2241) encoding a hygromycin B resistance gene ( $h p h)$ under the control of Aspergillus nidulans trpC promoter, and Zoanthus sp. green fluorescent protein $(Z s G F P)$ gene under the control of an $F$. verticillioides translation elongation factor 1- $\alpha$ promoter. The vector was transformed into Agrobacterium tumefaciens strain EHA105 by electroporation and by using random insertional mutagenesis for $V$. dahliae transformation. The putative GFP transformants were selected on PDA supplemented with hygromycin $(50 \mu \mathrm{g} / \mathrm{ml})$ and examined under a trinocular bright field/fluorescence microscope (Olympus BX60) equipped with a GFP filter (peak excitation wavelength at $489 \mathrm{~nm}$, peak emission wavelength at $508 \mathrm{~nm}$ ). The transformants were also examined for morphological features (colony growth and microsclerotia production) and pathogenicity on Long Purple eggplant, Early Pak tomato, and Salinas lettuce (data not presented) along with the wild-type strain. A transformed isolate with characteristics similar to the wild type was selected for inoculation followed by confocal microscopy.

\section{Sample collection for confocal microscopy}

Fourteen-day-old seedlings of Long Purple eggplant and Beefsteak tomato were inoculated with BCA PD659 using root dip, as described above. The GFP-tagged isolate Le1811 was inoculated 2 weeks postinoculation (wpi) with PD659 or sterile water treatment via soil drenching. At least three plants from each treatment were uprooted, washed free of soil, and used in confocal microscopy at 17 and 34 days after Le1811 inoculation. Several thin vertical or transverse sections were prepared from stem tissues sampled approximately $1 \mathrm{~cm}$ from the soil line to track pathogen growth within the plant. Crosssections were placed on a glass slide, covered with a glass coverslip number 1.5 (approximately $0.17 \mathrm{~mm}$ ), and observed under a confocal microscope (Leica LAS-AF V.3) at the Department of Plant Pathology, University of California, Davis. Default settings on the confocal microscope such as the acquisition of images (format $=512$ by 512 pixels, scan speed $=400 \mathrm{~Hz}$ ) and the channel settings were utilized. The GFP setting for the GFP signal (fluorescence emission maximum at $532 \mathrm{~nm}$ ) and autoflorescence of $680 \mathrm{~nm}$ was used. The captured digital images acquired were further processed using GIMP-2.10 (https://www.gimp.org/).

\section{RNA extraction and reverse-transcription quantitative real-time PCR}

To monitor plant defense responses in the presence or absence of BCA PD659, the expression of selected $P R$ marker genes was monitored using quantitative real-time PCR over time. Briefly, the susceptible Early Pak tomato was first inoculated with BCA PD659 or treated with water 14 days after germination using the root-dip method. Fourteen days after inoculation, seedlings from both sets were challenged with the $V$. dahliae isolate Le1811 from tomato using soil drench, as described above. The water-treated plants served as a negative control. Shoot tissues from all three treatment combinations (water, water followed by Le1811, and PD659 followed by Le1811) were collected using destructive sampling of actively growing tips of three plants per replication at $24 \mathrm{~h}, 48 \mathrm{~h}$, 1 week, and 2 weeks after inoculation of Le1811. Collected tissues were frozen immediately in liquid nitrogen and stored at $-80^{\circ} \mathrm{C}$ until use. In total, 36 samples (three treatments by four time points by three replications) were collected.

The reverse-transcription quantitative real-time PCR (RTqPCR) was performed following the method described by Puri et al. (2016). Briefly, total RNA was extracted using the SV Total RNA Isolation Kit (Promega Corp., Madison, WI, U.S.A.) and purified by treatment with DNase I (NEB, Ipswich, MA, U.S.A.) according to the manufacturers' protocols. An RT reaction was performed on $1 \mu \mathrm{g}$ of total RNA using SuperScript III reverse transcription (Invitrogen, Carlsbad, CA, U.S.A.). cDNA was diluted sixfold and used as a template for RT-qPCR using a LightCycler 480 II (Roche Life Science, Inc.). Three technical replicates were performed for each sample in $20-\mu 1$ reactions containing $2 \mu \mathrm{l}$ of cDNA template and $250 \mathrm{~nm}$ forward and reversed primers using SYBR Green PCR Master Mix (Applied Biosystems, Foster, CA, U.S.A.). PCR conditions included one cycle of preincubation at $50^{\circ} \mathrm{C}$ followed by $95^{\circ} \mathrm{C}$, each for $2 \mathrm{~min}$; then, 45 cycles of $95^{\circ} \mathrm{C}$ for $30 \mathrm{~s}$ and $59^{\circ} \mathrm{C}$ for $1 \mathrm{~min}$; followed by melt curve analysis. The differential expression of selected $P R$ genes was performed using previously standardized primer pairs. The primer sequences for tomato (Lycopersicon esculentum) acidic extracellular $\beta$-1,3-glucanase (GluA), basic intracellular $\beta$-1,3glucanase $(G l u c B)$, acidic extracellular chitinase (Chi3), basic intracellular chitinase (Chi9), and $P R$ gene SIPR5 were retrieved from Aimé et al. (2008) or Barda et al. (2015); PRla from Block et al. (2005); cysteine protease (cysProtease) from Balaji et al. (2008); and the reference gene actin from Herman et al. (2008). The constitutively expressed actin gene was used as a reference to calculate relative expression with respect to mock-inoculated samples using REST software, version 2009 (Pfaffl et al. 2002).

\section{Disease assessment and data analysis}

Disease severity (DS) was assessed at 8 to 10 wpi based on the discoloration of the vascular tissue of the taproot using a 0-to-5 subjective ordinal scale (Hayes et al. 2011b). Nonparametric analyses using PROC MIXED for analysis of variance (ANOVA)-type statistics of ranked mean, and LD_CI macro for relative marginal effects (RME) of each treatment at $95 \%$ confidence intervals (CI), were performed in SAS v9.4 (SAS Institute) (Shah and Madden 2004). Two treatments were 
considered significantly different when RME values had nonoverlapping CIs (Brunner and Langer 2000; Shah and Madden 2004). A higher RME value refers to higher DS. Orthogonal contrast analyses were used to compare the biocontrol effect of each treatment versus disease caused by the pathogenic isolate VdLs16. The Pearson Correlation Coefficients $(r)$ and correlograms among disease incidence, severity, plant height, and biomass were calculated using a ggscatter function in $\mathrm{R}$ package 'ggpubr.'

The biocontrol effect was quantified based on the decreased rate of disease (DRD) using the following formula: DRD = $\left(1-S_{i} / S_{m}\right) \times 100 \%$, where $S_{i}=$ the average DS of plants that received biocontrol treatment and $S_{m}=$ the average severity of plants inoculated with pathogenic isolates.

\section{RESULTS}

\section{Pathogenicity}

None of the biocontrol strains tested in the experiments caused any visible symptoms of Verticillium wilt in any hosts (Fig. 1).

\section{Biocontrol efficacy of $V$. klebahnii (PD659) and V. isaacii (PD660) against $V$. dahliae isolate VdLs16 across three hosts}

An ANOVA-type statistical analysis (ANOVA-F) using nonparametric methods showed significant differences among biocontrol treatments, host responses, and treatment $\times$ host interactions $(P<0.0001)$. The results from the two independent experiments were similar. Because experiments were not a significant source of variation $(P=0.6263)$ (Table 1$)$, the data from two independent experiments were combined for further analysis. PD659 against the $V$. dahliae isolate VdLs16 across the three hosts when introduced 2 weeks in advance of the pathogenic $V$. dahliae isolate VdLs16 (PD659-2w-VdLs16) (Fig. 1). This treatment, by far, had the lowest RME that was significantly different $(\mathrm{RME}=0.36, \mathrm{CI}=0.31$ to 0.41$)$ from other treatments, except when PD659 was introduced 1 week prior to the inoculations with $V$. dahliae isolate VdLs16 (PD659-1wVdLs16, RME $=0.44, \mathrm{CI}=0.37$ to 0.51 ) (Fig. 1). Treatments involving PD660 and T. flavus strain NRRL15936 also had significantly lower RME than VdLs16 alone, and all other treatments inoculated with VdLs16 prior to the inoculations with BCAs (Fig. 1). Overall, PD659 reduced Verticillium wilt severity the most relative to either PD660 or T. flavus when inoculated 2 weeks in advance of the pathogen. RME values were not significantly different between PD660 and T. flavus strain regardless of the treatment orders. All treatments where VdLs16 was introduced earlier than the biocontrol had

\section{TABLE 1}

Analysis of variance (ANOVA) using ANOVA F-type statistics of biocontrol treatments on the ranked mean of disease severity

\begin{tabular}{lrrrr}
\hline Effect & $\mathrm{df}_{\mathrm{N}}{ }^{\mathrm{a}}$ & $\mathrm{df}_{\mathrm{D}}$ & $F$ value & $P$ value \\
\hline Experiment & 1 & 659 & 0.24 & 0.6263 \\
Treatments & 19 & 659 & 266.10 & $<0.0001$ \\
Hosts & 2 & 659 & 41.95 & $<0.0001$ \\
Treatment $\times$ host & 38 & 659 & 230.20 & $<0.0001$ \\
\hline
\end{tabular}

${ }^{\mathrm{a}} \mathrm{df}_{\mathrm{N}}=$ numerator degree of freedom and $\mathrm{df}_{\mathrm{D}}=$ denominator degree of freedom.
The RME indicated a significant effect of BCA strain

consistently higher RME (0.60 to 0.68). Individual BCAs had the lowest RME (0.24 to 0.26$)$, followed by BCAs introduced 2 weeks $(0.36$ to 0.51$)$ and 1 week $(0.44$ to 0.55$)$ prior to inoculations with $V$. dahliae isolate VdLs16. Plants inoculated with VdLs16 had the highest (0.77) and significantly different RME than all other treatments $(\mathrm{CI}=0.67$ to 0.83 ) (Fig. 1$)$.

The median DS was zero for all BCAs (NRRL15936, PD659, and PD660), which was not significantly different than the water control in all hosts (Fig. 1). The median DS of treatments that received BCA as coinoculation, or BCA inoculated 1 and 2 weeks in advance of inoculations with VdLs16 was lower (2.0) than that caused by VdLs16 (Fig. 1). The orthogonal contrasts between VdLs16 versus all other possible treatment combinations were highly significant $(P<0.001)$, except for VdLs16 versus VdLs16-1wk-PD659 $(P=0.1697)$ and VdLs16 versus VdLs16-2wk-PD659 $(P=0.091)$ (Table 2). These results provide additional evidence that the introduction of any of the BCAs (i.e., PD659, PD660, or T. flavus strain NRRL15936) as either coinoculation or 1 and 2 weeks in advance of the pathogenic strain significantly reduce Verticillium wilt caused by the pathogenic strain.

\section{Biocontrol efficacy on lettuce, tomato, and eggplant against VdLs16}

The BCAs V. klebahnii (PD659), V. isaacii (PD660), and T. flavus (NRRL15936) on individual hosts (i.e., eggplant, lettuce, and tomato) showed a host-dependent disease suppression of Verticillium wilt caused by VdLs16. Of these, PD659 had higher efficacy than either PD660 or T. flavus strain NRRL15936 in all hosts (Fig. 2). In eggplant, PD659 inoculated 2 weeks before VdLs16 had the highest DRD $(82.0 \%)$, followed by 1 week $(72.9 \%)$ and coinoculation $(63.3 \%)$, and also had consistently higher efficacy in lettuce (100\%) and tomato (61.1\%) (Fig. 2).

\section{TABLE 2}

Orthogonal contrasts of Verticillium dahliae isolate VdLs16 versus biocontrol treatments combined across the three hosts

\begin{tabular}{lcccc}
\hline Contrasts & $\mathrm{df}_{\mathrm{N}}{ }^{\mathrm{a}}$ & $\mathrm{df}_{\mathrm{D}}$ & $F$ value & $P$ value \\
\hline VdLs16 versus PD659 & 1 & 659 & 308.44 & $<0.0001$ \\
VdLs16 versus PD660 & 1 & 659 & 273.97 & $<0.0001$ \\
VdLs16 versus NRRL15936 & 1 & 659 & 230.15 & $<0.0001$ \\
VdLs16 versus PD659 + VdLs16 & 1 & 659 & 21.8 & $<0.0001$ \\
VdLs16 versus PD660 + VdLs16 & 1 & 659 & 18.05 & $<0.0001$ \\
VdLs16 versus & 1 & 659 & 30.57 & $<0.0001$ \\
$\quad$ NRRL15936 + VdLs16 & & & & \\
VdLs16 versus PD659-1wk-VdLs16 & 1 & 659 & 20.27 & $<0.0001$ \\
VdLs16 versus PD660-1wk-VdLs16 & 1 & 659 & 39.52 & $<0.0001$ \\
VdLs16 versus & 1 & 659 & 31.92 & $<0.0001$ \\
$\quad$ NRRL15936-1wk-VdLs16 & & & & \\
VdLs16 versus PD659-2wk-VdLs16 & 1 & 659 & 50.5 & $<0.0001$ \\
VdLs16 versus PD660-2wk-VdLs16 & 1 & 659 & 18.05 & $<0.0001$ \\
VdLs16 versus & 1 & 659 & 57.31 & $<0.0001$ \\
$\quad$ NRRL15936-2wk-VdLs16 & & & & \\
VdLs16 versus VdLs16-1wk-PD659 & 1 & 659 & 1.89 & 0.1697 \\
VdLs16 versus VdLs16-1wk-PD660 & 1 & 659 & 9.42 & 0.0022 \\
VdLs16 versus & 1 & 659 & 12.25 & 0.0005 \\
$\quad$ VdLs16-1wk-NRRL15936 & & & & \\
VdLs16 versus VdLs16-2wk-PD659 & 1 & 659 & 2.68 & 0.091 \\
VdLs16 versus VdLs16-2wk-PD660 & 1 & 659 & 8.47 & 0.0037 \\
VdLs16 versus & 1 & 659 & 4.55 & 0.0332 \\
$\quad$ VdLs16-2wk-NRRL15936 & & & & \\
\hline a df $=$ numerator degree of freedom and $\mathrm{df}_{\mathrm{D}}=$ denominator & degree of \\
freedom. & & & &
\end{tabular}


In eggplant, the disease suppression by PD659 was higher than by PD660 regardless of the inoculation treatments. Similarly, disease suppression by PD659 was higher than by $T$. flavus strain NRRL15936, except when this strain was coinoculated with VdLs16 that suppressed Verticillium wilt to a higher degree than PD659 (70.8 versus 63.3\%). In lettuce, the efficacy of PD659 (100\%) was significantly higher than either with PD660 (53.5\%) or T. flavus strain NRRL15936 $(79.5 \%)$ when inoculated 2 weeks in advance of VdLs16. Although the overall efficacy of all BCAs used were lower in tomato, PD659 still had higher efficacy (37.6 to 60.1\%) than PD660 (27.9 to 37.0\%) and T. flavus strain NRRL15936 (25.5 to $27.9 \%$ ) (Fig. 2).

Likewise, $V$. isaacii strain PD660 also showed a higher biocontrol efficacy on eggplant and lettuce than in tomato against VdLs16. In lettuce, disease reduction was $97.5 \%$ when PD660 was inoculated 1 week in advance of VdLs16, which was even higher than with PD659 $(60.9 \%)$ for the same treatment. Similarly, DRD with PD660 was higher than with PD659 in coinoculation treatments (51.7 versus $16.9 \%)$. A comparable biocontrol efficacy was observed between PD660 and T. flavus strain NRRL15936 in all three hosts, except the coinoculation treatment in tomato and inoculation of PD660 1 week prior in lettuce, which had significantly higher efficacy than other combinations (Fig. 2).

The standard BCA T. flavus strain NRRL15936 alone ensured consistently higher biocontrol efficacy (42.3 to $79.5 \%$ ) in eggplant and lettuce for all three inoculation treatments (coinoculation, 1 week, and 2 weeks in advance of inoculations with VdLs16) than in tomato (22.6 to $27.9 \%$ ). It had significantly high efficacy when coinoculated in eggplant $(70.8 \%)$ and preinoculated 2 weeks prior in lettuce $(79.5 \%)$. All BCAs had at least $16.9 \%$ DRD regardless of the hosts or time of BCA inoculation (Fig. 2). However, inoculations of VdLs16 before the BCAs resulted in very low or nonsignificant DRD (data not shown).

\section{Biocontrol efficacy of PD659 and PD660 on eggplant, lettuce, and tomato against corresponding isolates from each host}

The biological control efficacy of $V$. klebahnii (PD659) and $V$. isaacii (PD660) was further confirmed by challenging each host with an isolate from the corresponding host. The ANOVA indicated highly significant differences among the biocontrol treatments in each host $(P<0.001)$ (Table 3). Plants inoculated with BCAs developed no Verticillium wilt symptoms aboveground and vascular tissue discoloration on the taproots.

Biocontrol efficacy against Sm113 in eggplant. Both PD659 and PD660 reduced Verticillium wilt in eggplant challenged with the eggplant isolate Sm113 (Fig. 3). Plants inoculated with Sm113 alone exhibited clear Verticillium wilt symptoms such as severe stunting, dried leaves that curled upwards, and

TABLE 3

Nonparametric analysis of variance (ANOVA) statistics on Verticillium wilt responses on biocontrol treatments to host-dependent isolates

\begin{tabular}{lcccc}
\hline & \multicolumn{4}{c}{ ANOVA-type statistic } \\
\cline { 2 - 5 } Treatment effects & $\mathrm{df}_{\mathrm{N}}{ }^{\mathrm{a}}$ & $\mathrm{df}_{\mathrm{D}}$ & $F$ & $P$ value \\
\hline Eggplant & 2.76 & 55.7 & 61.55 & $<0.0001$ \\
Lettuce & 7.37 & 154 & 16.74 & $<0.0001$ \\
Tomato & 2.83 & 153 & 247.7 & $<0.0001$ \\
\hline
\end{tabular}

${ }^{a} \mathrm{df}_{\mathrm{N}}=$ numerator degree of freedom and $\mathrm{df}_{\mathrm{D}}=$ denominator degree of freedom.

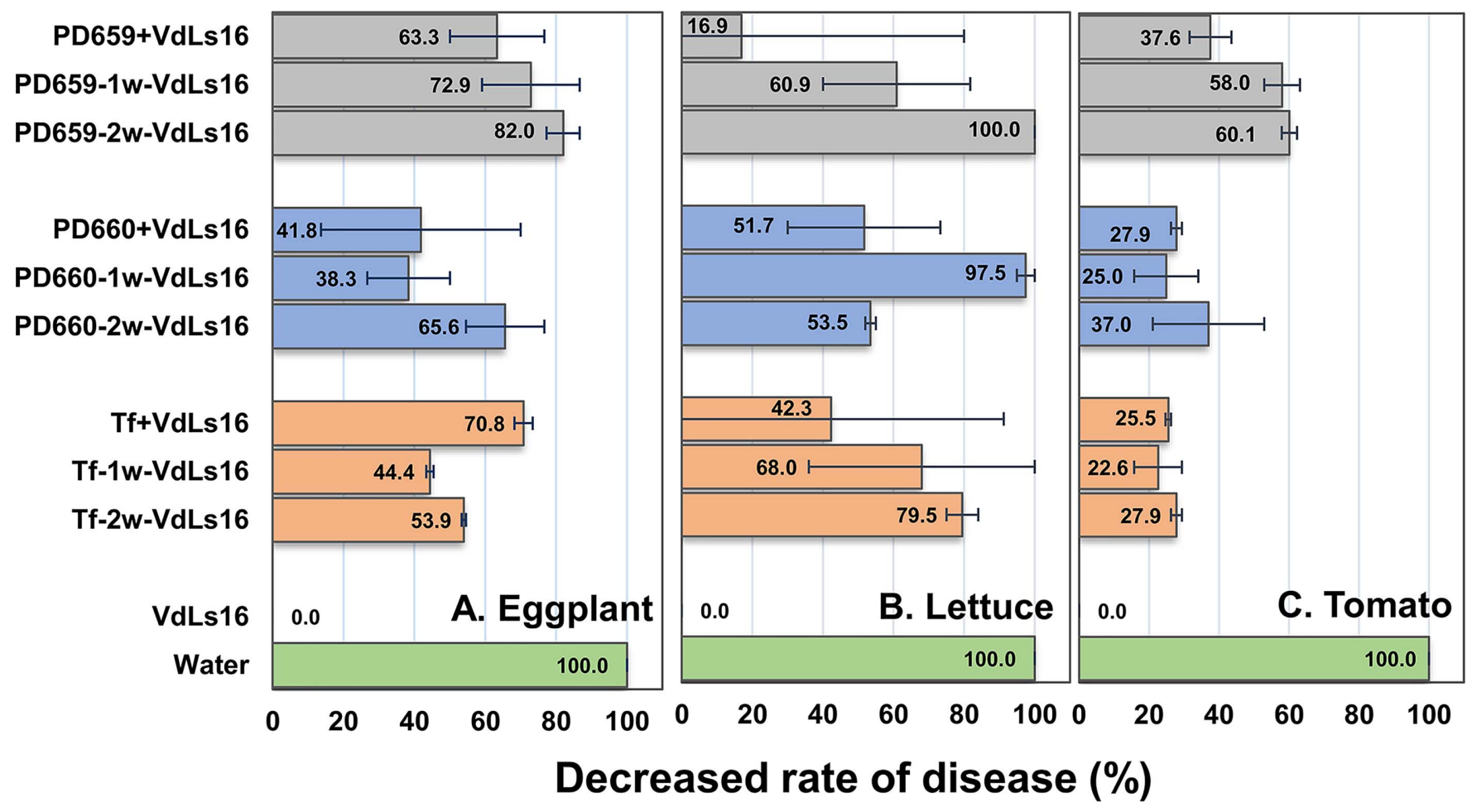

FIGURE 2

Biocontrol efficacy of Verticillium klebahnii (isolate PD659), V. isaacii (isolate PD660), and Talaromyces flavus (strain NRRL15936) evaluated as the decreased rate of disease on A, eggplant; B, lettuce; and C, tomato caused by the V. dahliae isolate (VdLs16) from lettuce. The horizontal line inside the bar represents the standard error of the mean from two independent experiments. 
extensive vascular discoloration of the taproot and stem compared with the treatments that received PD659, PD660, or water (Fig. 3A). Plant were significantly taller in BCA treatments than plants inoculated with Sm113 and Water-2w-Sm113 inoculation but significantly lower than in uninoculated controls (Fig. 3B). The RME values for both biocontrol strains were significantly lower than the pathogenic isolates inoculated at either 2 weeks (Sm113) or 4 weeks (Water-2w-Sm113) (Fig. 3C). The differences between PD659 and PD660 $(P<0.05)$ for RME or plant height were not significant.

Plant height had highly significant positive correlation to total plant biomass (root + shoot $)(r=0.93, P<0.001)$ (Fig. 4A). Both biomass and plant height were negatively correlated with DS (Fig. 4BE) and disease incidence (Fig. 4CF). However, a highly significant positive correlation existed between DS and disease incidence $(r=0.97, P<0.001)$ (Fig. 4D), suggesting that either variable can be used to assess the effectiveness of treatments.

Biocontrol efficacy against VdLs16 and VdLs17 in lettuce. Consistent with the previous experiments, lettuce inoculated with $V$. dahliae isolates VdLs16 (race 1) and VdLs17 (race 2) exhibited foliar wilt symptoms and taproot vascular tissue discoloration in susceptible Salinas lettuce. The VdLs17inoculated plants had a higher RME than those inoculated with VdLs16 but values were not significantly different $(P<0.05)$. Even though RME values for the different treatments were varied, they were not significantly different from each other when inoculated with VdLs17 $(P<0.05)$. Both PD659 $(\mathrm{RME}=0.37)$ and PD660 $(\mathrm{RME}=0.36)$ had significantly lower DS and RME values than the VdLs16-positive control when inoculated on 2-week-old seedlings (VdLs16, $\mathrm{RME}=0.65$ ) but not for seedlings inoculated at 4 weeks (Water-2w-VdLs16, RME = 0.40). In the case of VdLs17, the DS was reduced moderately when BCAs were applied 2 weeks before VdLs17 inoculation but the RME was not significantly different between control and treated plants (Fig. 5).

Biocontrol efficacy against Le1811 in tomato. Early Pak tomato inoculated with the tomato $V$. dahliae isolate Le1811 developed Verticillium wilt symptoms, including plant stunting, chlorosis, and wilting of lower leaves (Fig. 6A). Vascular tissue discoloration of the taproot and stem was also observed. In general, a more prominent plant canopy and significantly taller plants were associated with both BCAs (PD659 and PD660) than with Le1811, though they weren't significantly different from the Water-2w-Le1811 treatment (Fig. 6B). The RME values indicated a significant reduction in Verticillium wilt severity by both BCAs regardless of the time of pathogenic isolate inoculation (Fig. 6C).
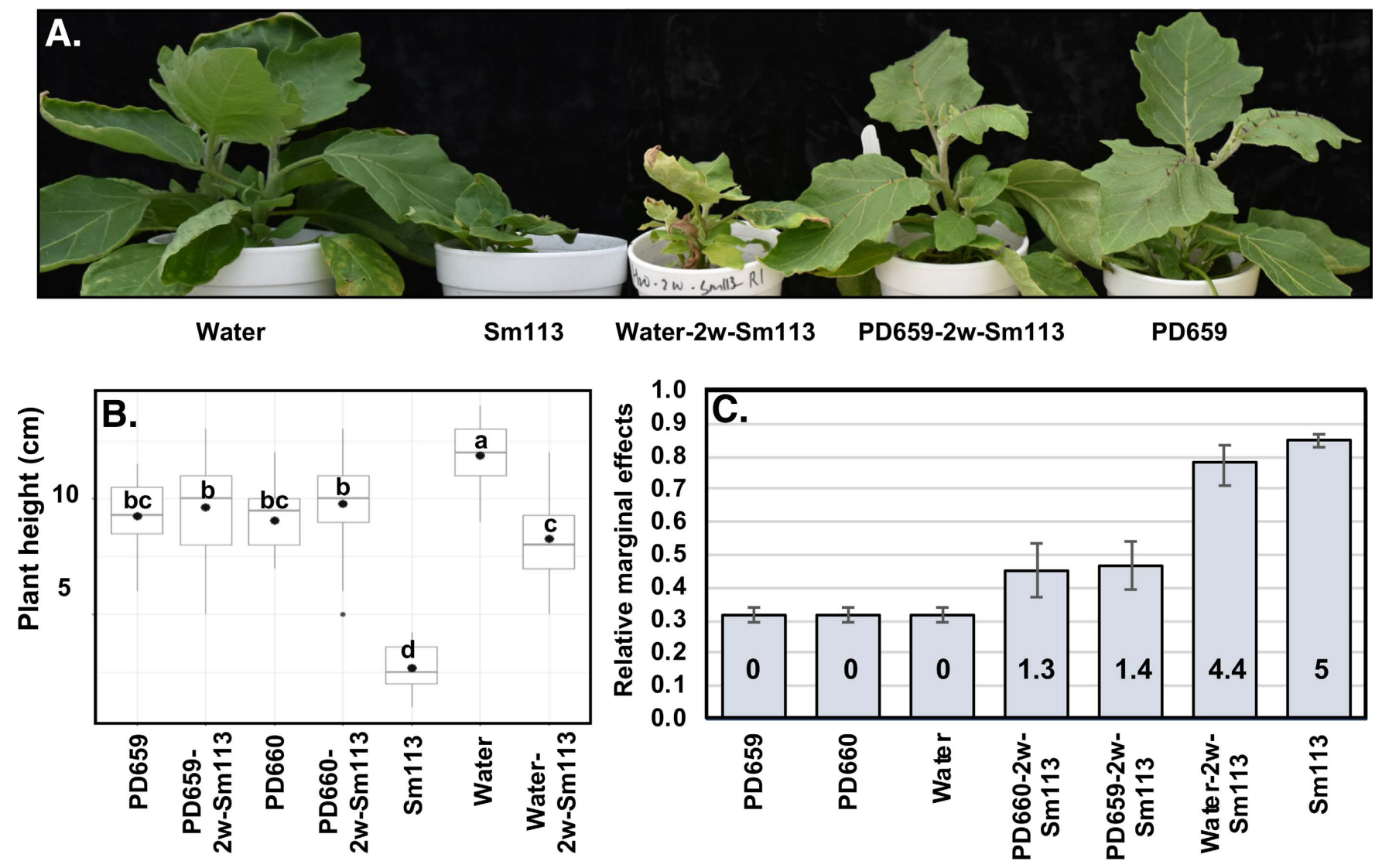

FIGURE 3

Biological control effect of Verticillium klebahnii (PD659) and V. isaacii (PD660) against V. dahliae isolate Sm113 from the eggplant. A, Morphological changes; B, plant height; and C, relative marginal effect (RME) (numbers inside the bars represent mean disease severity) and in response to various treatment combinations were shown. RME was calculated using LD_Cl macros (Shah and Madden 2004) in SAS 9.4. Pictures were taken at the time of disease rating at approximately 8 weeks postinoculation with the pathogenic isolate. Four-week-old seedlings were inoculated with pathogenic isolates Sm113 that received either biocontrol (PD659 or PD660) or water treatment at 14 days after germination. Seedlings were 2 weeks old at the time of inoculations with PD659, PD660, and Sm113 but were 4 weeks old in all other treatments. 


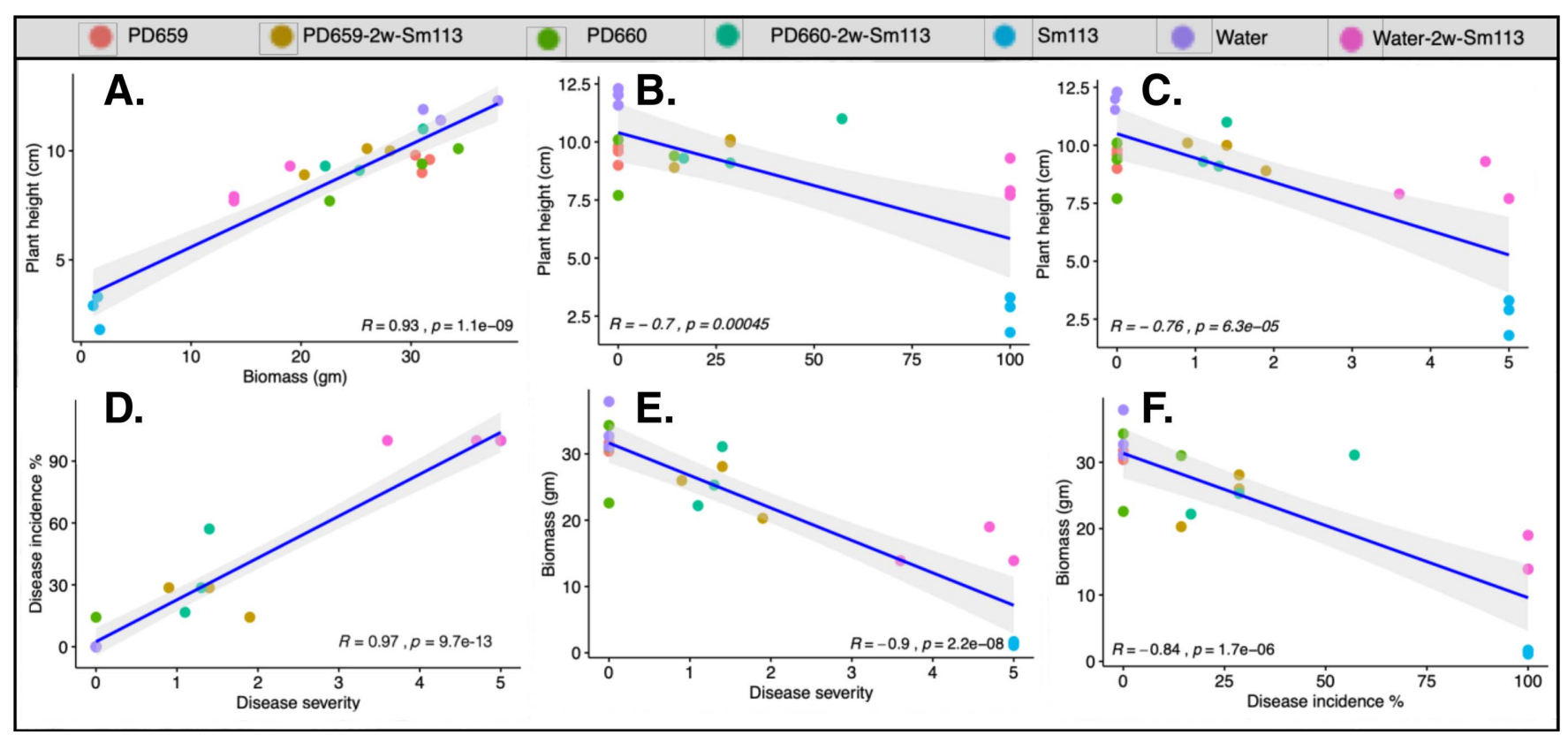

FIGURE 4

Correlation among plant height, biomass, disease severity, and disease incidence of all treatments in eggplant. Four-week-old seedlings were inoculated with isolate Sm113 that had received either biocontrol (PD659 or PD660) or water treatment at 14 days after germination. A, Biomass versus plant height; B, disease severity versus plant height; C, disease incidence versus plant height; $\mathbf{D}$, disease severity versus disease incidence; $\mathbf{E}$, disease severity versus biomass; and $\mathbf{F}$, disease incidence versus biomass.

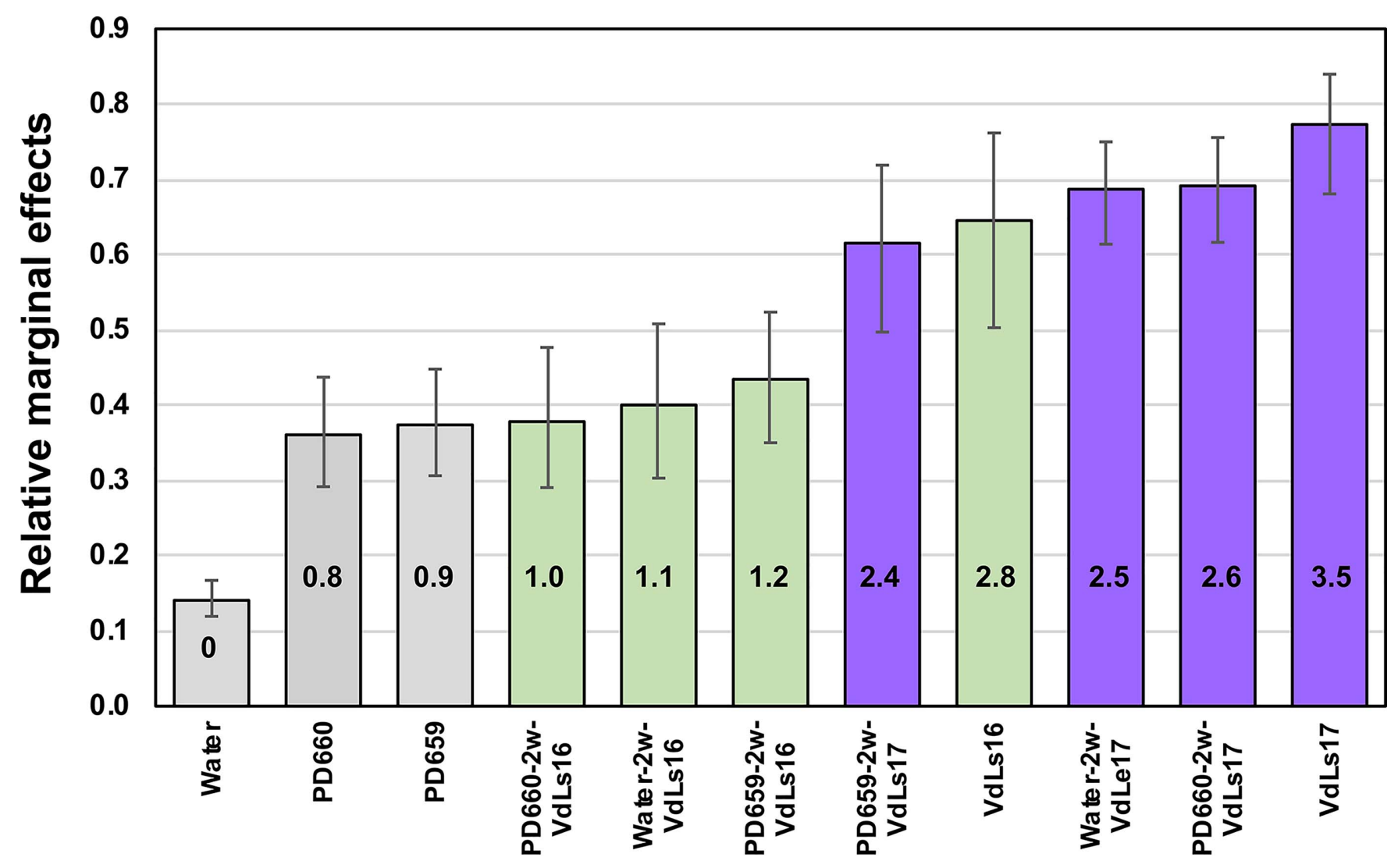

FIGURE 5

Relative marginal effect (RME) values of Verticillium klebahnii (PD659) and V. isaacii (PD660) against isolates VdLs16 and VdLs17 from lettuce. RME values were calculated using LD_CI macros (Shah and Madden 2004) in SAS 9.4. Numbers inside the bars represent mean disease severity. Both biocontrol and control isolates were first inoculated to 2-week-old seedlings using root dipping. Two weeks after inoculation (4-week-old seedlings), pathogenic isolates VdLs16 and VdLs17 were inoculated to seedlings that received either biocontrol (PD659 or PD660) or water treatment at 2 weeks old. Seedlings were 2 weeks old at the time of inoculations with PD659, PD660, VdLs16, or VdLs17 but were 4 weeks old in all other treatments. 


\section{Confocal microscopy of $\boldsymbol{V}$. dahliae progress on eggplant and tomato}

Pathogen colonization in the vascular systems of eggplant and tomato following inoculations of the GFP-tagged $V$. dahliae in the presence or absence of BCA $V$. klebahnii PD659 were determined by confocal microscopy. In eggplant, at 17 days postinoculation (dpi), the colonization of vascular tissue at the stem base (1 cm above the soil line) was visible slightly in the presence of biocontrol (Fig. 7A) compared with the visibly higher vascular tissue colonization in treatments without BCA (Fig. 7B). However, at 34 dpi, vascular tissue colonization levels were indistinguishable between the treatments (Fig. 7C and D). In tomato, however, no visible colonization of the vascular tissues was evident in the BCA-treated plants (Fig. 7E). In contrast, a moderate level of colonization was distinct in the absence of biocontrol (Fig. 7F) at 17 DAI. A similar trend occurred at 34 DAI, where only a few colonized vessels were observed in
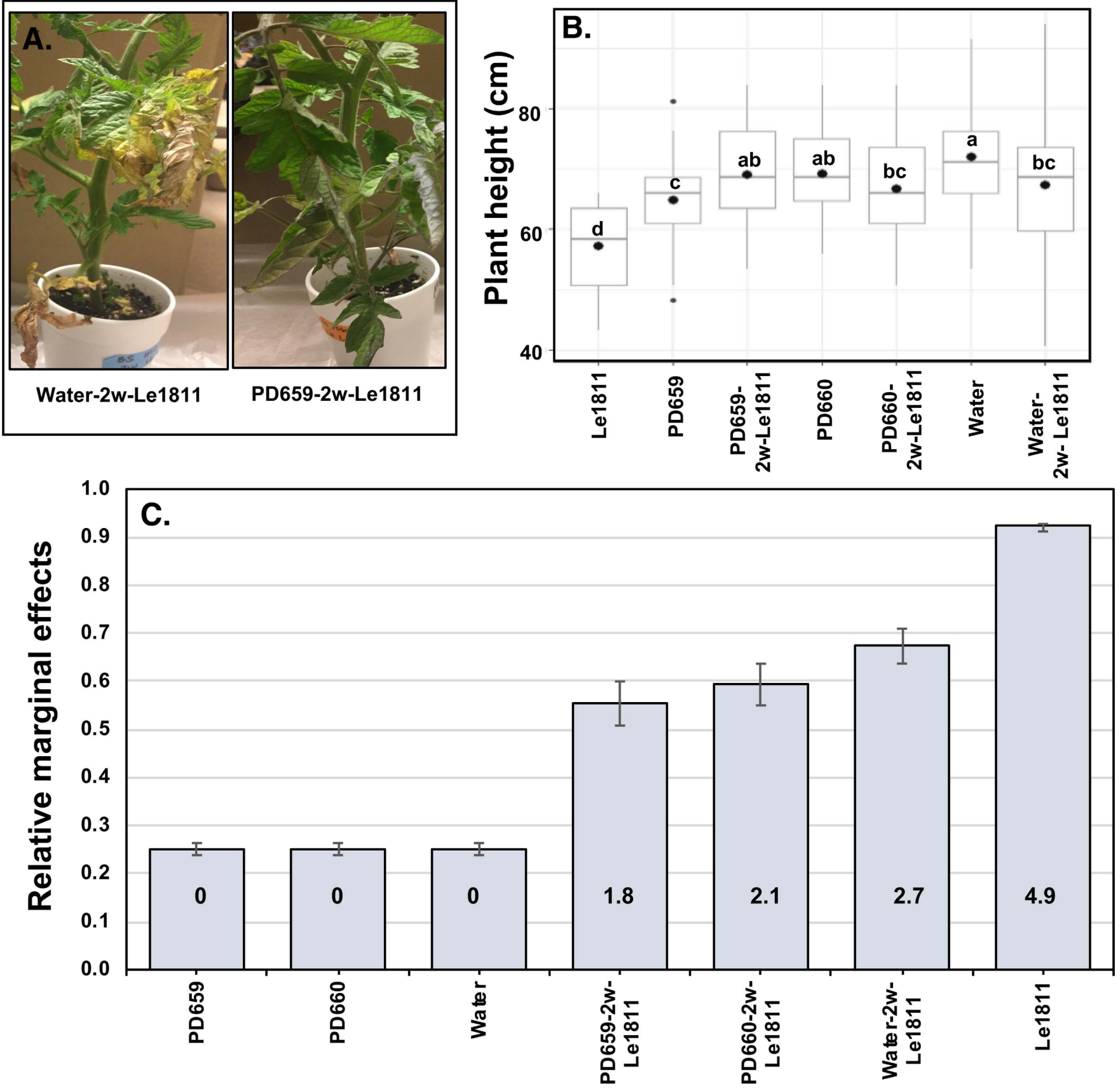

FIGURE 6

Biological control effect of Verticillium klebahnii (PD659) and V. isaacii (PD660) against isolate Le1811 from tomato. A, Morphological changes; B, plant height; and C, relative marginal effects (RME) (numbers inside the bars represent mean disease severity). RME were calculated using LD_CI macros (Shah and Madden 2004) in SAS 9.4. Confidence intervals for PD659-2w-Le1811, PD660-2w-Le1811, and Water-2w-Le1811 treated with the two biocontrol agents were 0.5079 and $0.5999,0.5501$ and 0.6367 , and 0.6369 and 0.7095 , respectively, while for Le1811 they were 0.9119 and 0.9275 . Pictures were taken at the time of disease rating at approximately 8 weeks postinoculation of the pathogenic isolate. Four-week-old-seedlings were inoculated with pathogenic strain Le1811 that received either biocontrol (PD659 or PD660) or water treatment at 14 days. 
BCA-treated plants (Fig. 7G), whereas the vascular tissue was extensively colonized in plants inoculated with the Le1811 isolate alone (Fig. 7H).

\section{Altered expression of defense response genes to biocontrol treatments}

The putative BCA $V$. klebahnii isolate PD659, when inoculated 2 weeks in advance of the pathogen, prevented recognition of the pathogen, as indicated by the lack of induced resistance gene expression in biocontrol-treated tomato plants. None of the genes evaluated (i.e., PRla, PR5, GlucA, GlucB, Chi3, Chi9, and cysProtease) had significant expression or upregulation in biocontrol-treated plants at all time points ( $24 \mathrm{~h}$ postinoculation [hpi] to $2 \mathrm{wpi}$ ) relative to the expression in mock-inoculated plants (Fig. 8).

However, in plants inoculated with the pathogenic $V$. dahliae isolate Le1811, significant upregulation of the PR genes (i.e., PRla, PR5, GlucA, GlucB, Chit3, Chit9, and cysProtease) at various time points relative to their expression in water-treated plants was observed (Fig. 8). PRla was the most highly upregulated gene at 24 hpi $(18.8$-fold, $P<0.01)$ and 48 hpi (19.6fold, $P=0.086$ ), followed by $P R 5$ (7.4-fold, $P=0.067$ ) and GlucB (10.7-fold, $P<0.05$ ), respectively. However, the differences in expression were not significant at 1 or 2 wpi, except for $P R 5$ and GlucA, which exhibited increased expression in response to Le1811 at 2 wpi $(P<0.05)$ (Fig. 8).

\section{DISCUSSION}

The populations of $V$. dahliae were predominantly composed of race 1 and race 2 in lettuce and tomato, until recently. A third race was identified in tomato from Japan (Usami et al. 2017). Resistance against $V$. dahliae race 1 is available in several hosts, including lettuce and tomato (Alexander 1962; Hayes et al. 2007), and against race 2 only in wild tomato species Solanum neorickii (Usami et al. 2017). Partial resistance, however, is available against it in lettuce (Sandoya et al. in press). Despite the availability of resistance against race 1 in lettuce, no commercial cultivars with resistance have been deployed. The phase-out of widely used soil fumigant MB left few effective chemical alternatives for disease management (Duniway 2002; Meadows 2013). This calls for the integration of complementary management options. BCAs could be a dependable alternative for managing $V$. dahliae (Fravel 2005) provided locally adapted strains with efficacy are identified. Therefore, we assessed one isolate each from $V$. klebahnii (PD659) and $V$. isaacii (PD660) for their biocontrol potential against Verticillium wilt caused by $V$. dahliae in eggplant, lettuce, and tomato. We determined that the nonpathogenic $V$. isaacii (PD660) and V. klebahnii (PD659) isolates protected crops against virulent strains of $V$. dahliae in a host-dependent manner, with higher efficacy when inoculated in advance of the pathogenic strain. Previous studies have also demonstrated biocontrol efficacy of certain

\section{Eggplant- Long Purple}
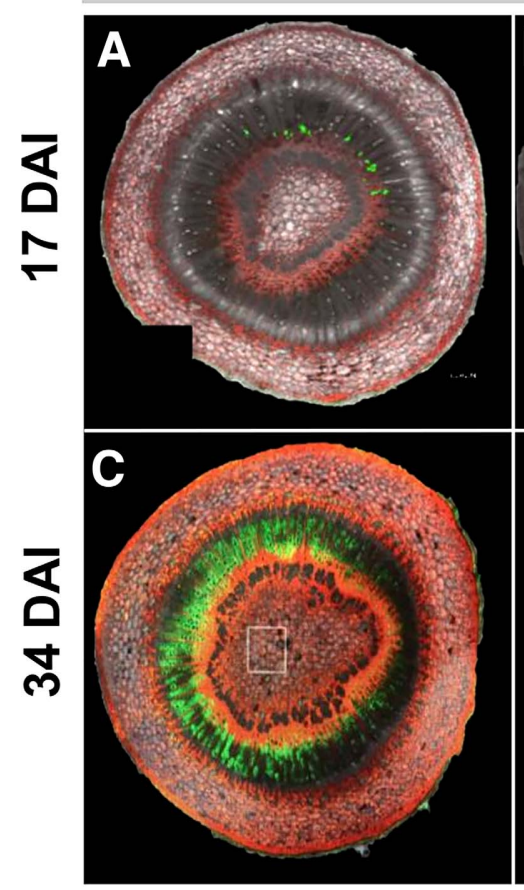

PD659-2w-Le1811
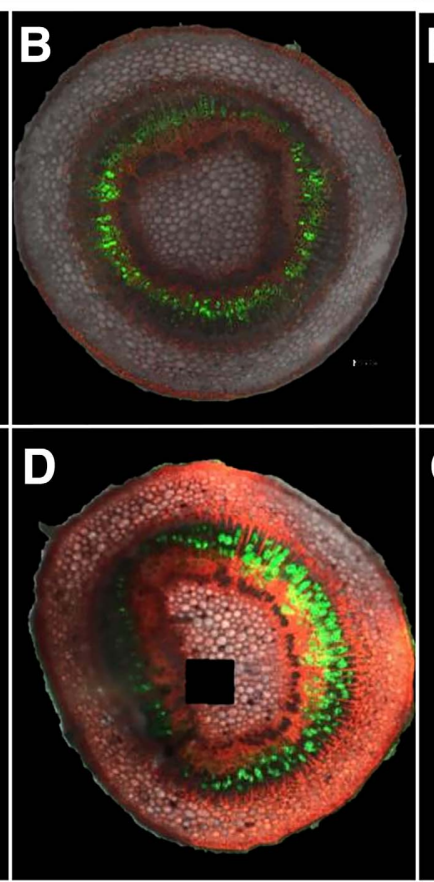

Water-2w-Le1811

\section{Tomato- Beefsteak}
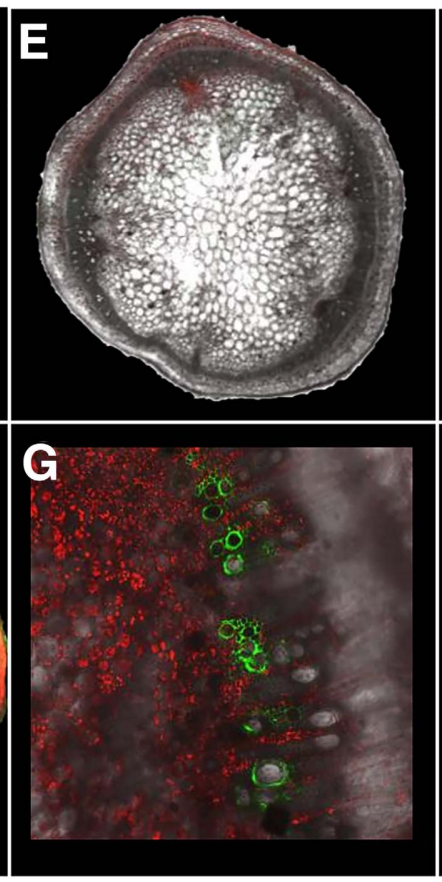

PD659-2w-Le1811

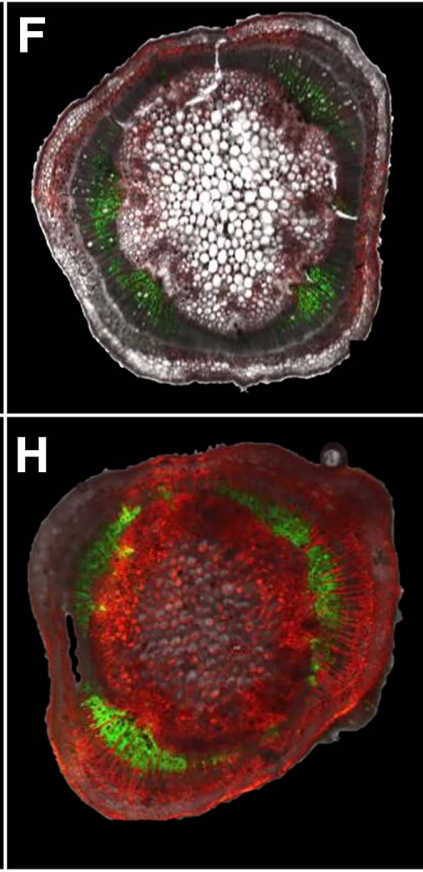

Water-2w-Le1811

FIGURE 7

Transverse sections of eggplant and tomato stem colonized by a green fluorescent protein (GFP)-expressing isolate of Verticillium dahliae Le1811 in the presence or absence of biocontrol treatments. The stem base at $1 \mathrm{~cm}$ above the soil line was sampled at 17 (upper) and 34 (lower) days after inoculation (DAI) with the GFP-tagged isolate for imaging. Biocontrol treatments received PD659 inoculation on 2-week-old seedlings followed by inoculations with the GFP-tagged isolate Le1811 after 14 days on A and C, Long Purple eggplant and $\mathbf{E}$ and $\mathbf{G}$, Beefsteak tomato. Controls $\mathbf{B}$ and $\mathbf{D}$, eggplant and $\mathbf{F}$ and $\mathbf{H}$, tomato were first inoculated with water followed by the pathogenic isolate at the same time as biocontrol agent-inoculated plants received the GFP-tagged isolate Le1811. Imaging was done using a Lecia confocal laser-scanning microscope at 10x, where Tile Scan Acquisition Mode was used for all images except for $\mathrm{H}$. 
strains of $V$. tricorpus and $V$. isaacii (formerly $V$. tricorpus) in Verticillium wilt suppression of eggplant, lettuce, cauliflower, and potato via endophytism, cross-protection, and induced resistance (Davis et al. 2000; Qin et al. 2008; Robinson et al. 2007; Tyvaert et al. 2014). The known fungal antagonist T. flavus, which was used as a reference BCA in the present study, also exhibits hyperparasitism to a broad spectrum of soilborne pathogens or directly kills microsclerotia of $V$. dahliae (Fravel 1989; Marois et al. 1982, 1984; Nagtzaam et al. 1998; Yuan et al. 2017; Zeise and Kersten 2000). Thus, the higher or comparable efficacy of PD659 and PD660 relative to T. flavus strain NRRL15936 positions them as potentially valuable BCAs for Verticillium wilt management. These strains are much better adapted to the agricultural ecosystems from which they were isolated and would not encounter the problems of a BCA that is unadopted to the system.

Although it was not possible to track the colocalization of pathogenic and BCA strains on the root surface or inside the roots during infection, it is evident that these BCAs excluded the pathogenic strains by competing for the same ecological niche, space, and nutrients in a host-dependent manner. A significantly higher biocontrol efficacy against host-dependent isolates in eggplant and tomato relative to lettuce is likely due to host-specific endophytic behavior of these BCAs on different hosts (Malcolm et al. 2013; Tyvaert et al. 2014). In lettuce, the pathogenic strain may take advantage of dense feeder root architecture that has not been entirely occupied by BCAs and initiate infection at later stages. Qin et al. (2008) demonstrated variability in biocontrol efficacy and suggested specificity of nonpathogenic $V$. tricorpus isolates VdLs432, VdLs441, and VdLs443 in lettuce. These isolates, which were subsequently reclassified as $V$. isaacii (Inderbitzin et al.
2011), were highly effective in reducing DS caused by V. dahliae isolate VdLs17 but not VdLs16 (Qin et al. 2008). The infection of $V$. isaacii isolates was dependent on the crop species and cultivars (Gurung et al. 2015). The $V$. isaacii isolate Vt305 (previously classified as $V$. tricorpus) from cauliflower effectively reduced the DS caused by $V$. longisporum in cauliflower (Tyvaert et al. 2014). Other known fungal antagonists (i.e., genera Trichoderma, Talaromyces, or bacterial endophytes such as Bacillus, Pseudomonas, and so on) were effective in providing cross-protection against various soilborne pathogens, including $V$. dahliae, Rhizoctonia solani, and Sclerotinia sclerotiorum, in a host-dependent manner (Eljounaidi et al. 2016; Madi et al. 1997). Therefore, the interactions between different Verticillium BCAs and host species have played a role in determining their efficacy. The disparity in the effectiveness of these BCAs in the first and second experiment in lettuce was likely due to the age of seedlings when they first received the pathogen. In the first experiment, comparisons were made with lettuce seedlings that were inoculated with the pathogen at 2 weeks after germination while, in the second experiment, seedlings were 4 weeks old when they were inoculated. This interaction signifies that the infection establishment occurs more efficiently and rapidly on younger seedlings than in older seedlings and likely plays a critical role in the overall disease progression. Thus, seedling transplanting rather than direct seeding may be an alternative strategy to reduce Verticillium wilt in lettuce fields with a history of consistently high Verticillium wilt incidence.

Furthermore, the efficacy of BCAs against soilborne plant pathogens largely depends on application timing and delivery mechanism (Angelopoulou et al. 2014; El-Hassan and Gowen 2006). We found a significantly high efficacy of PD659 when
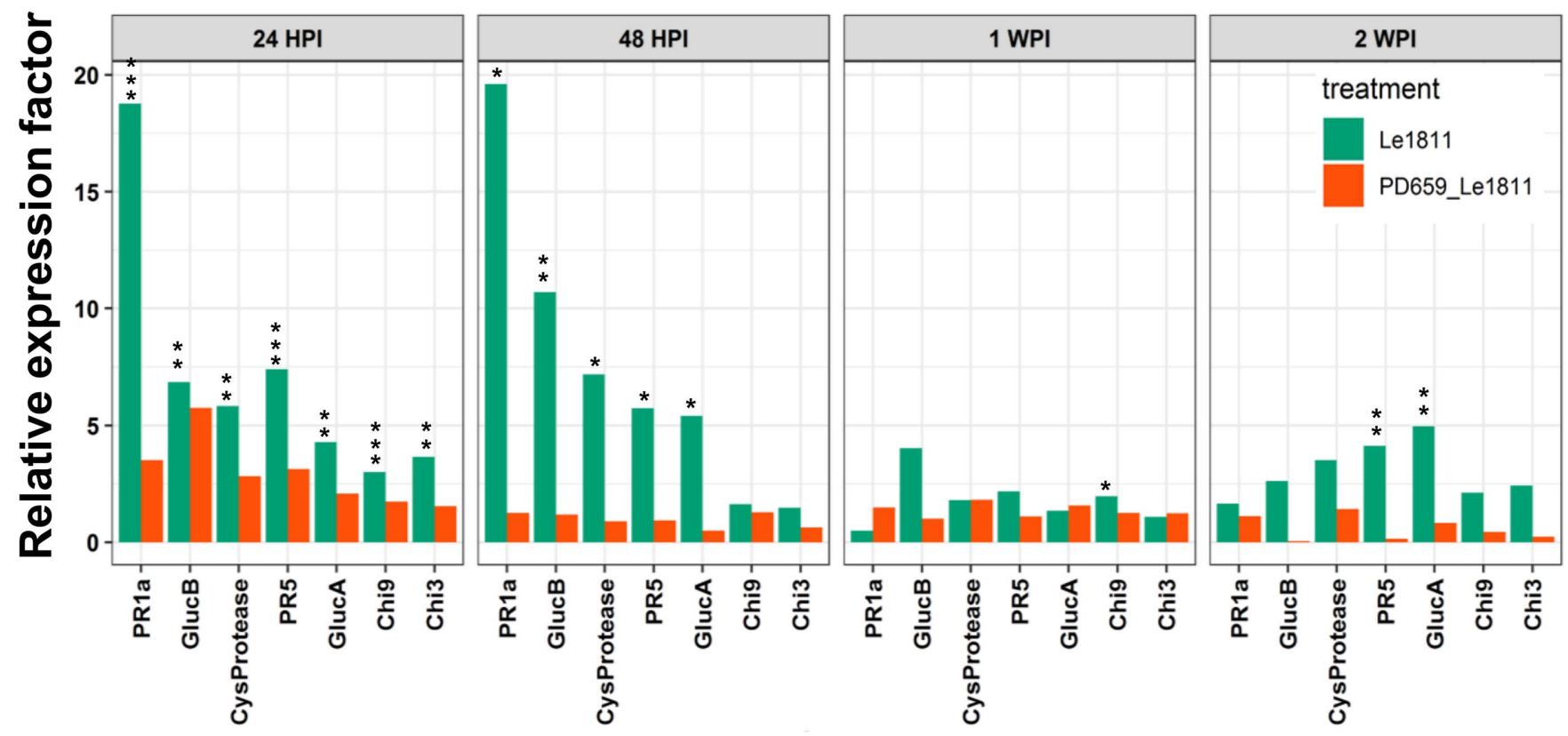

Target genes

FIGURE 8

Relative expression of defense response genes in Early Pak tomato in the presence and absence of biocontrol agents (BCAs) and normalized with tomato actin as the reference gene. BCA PD659 was inoculated 2 weeks before pathogenic Verticillium dahliae isolate Le1811. Leaf tissues were harvested at 24 and $48 \mathrm{~h}$ after pathogen inoculation (HPI) and 1 and 2 weeks after pathogen inoculation (WPI) on BCA or water-treated plants. Mean expression and error was determined using the relative expression software REST (Pfaffl et al. 2002). Quantitation cycle values from three biological replicates, each combined from three technical replications, were examined. Genes at least twofold upregulated and significant at $P<0.09,0.05$, and 0.001 are indicated by *, **, or ***, respectively. 
introduced 2 weeks in advance of the pathogenic isolates in the greenhouse. A similar interaction was observed in vitro when endophytic strain Dvd-E6 inoculated before or together with the pathogenic $V$. dahliae strain $\mathrm{Vd} 1$ and led to the complete exclusion of pathogenic strain from tomato roots (Shittu et al. 2009). Qin et al. (2008) observed better protection against Verticillium wilt of lettuce when individual isolates of $V$. isaacii were inoculated 1 week in advance of the pathogenic strain rather than a coinoculated treatment. Preinoculation of a nonpathogenic $V$. albo-atrum group 2 isolate 4 days prior to the pathogenic $V$. tricorpus reduced wilt severity more than the simultaneous inoculation of both in potato (Robinson et al. 2007). Other fungi such as Gibellulopsis nigrescens (reclassified from Verticillium by Zare et al. [2007]) isolate $\mathrm{CVn}-\mathrm{WHg}$, and nonpathogenic $V$. dahliae isolate $\mathrm{CVd}-\mathrm{WHw}$ reduced Verticillium wilt incidence significantly $(77.9$ to $95 \%)$ when inoculated 4 days before a highly virulent $V$. dahliae isolate CVd-AYb in cotton (Zhu et al. 2013). V. albo-atrum strain WCS850 effectively reduced the Dutch elm disease and is now available as a commercial formulation for injection into tree trunks (Postma and Goossen-van de Geijn 2016).

When direct seeding is not possible, lettuce is generally transplanted into the fields in coastal California. Thus, the introduction of these BCAs during transplant production in seeding trays at least 2 weeks prior to transplanting likely protects plants against Verticillium wilt in the field. Seed coating of beneficial microbes in vegetables and other crops is widely practiced to promote plant vigor and protect against many diseases (Rocha et al. 2019), including $V$. dahliae (Müller and Berg 2008) and V. longisporum (Deketelaere et al. 2020). Integration of Bacillus subtilis as seed treatment with carriers such as glucose and peat provided better disease suppression against $F$. oxysporum f. sp. lentils (El-Hassan and Gowen 2006). Thus, it is worth investigating the possibility of developing a seed-priming technique using PD659 in lettuce.

Instead of a universal mechanism by which nonpathogenic isolates of Verticillium spp. protect against pathogenic $V$. dahliae strains in all systems, there could be multiple modes of action. We observed a significant increase in plant biomass and plant height that directly correlated with low disease incidence and DS in the presence of BCAs. The reduced Verticillium wilt severity in tomato, eggplant, and other solanaceous crops was associated with increased plant vigor due to increased nitrogen and phosphorous uptake after using arbuscular mycorrhizal fungus (Demir et al. 2015; Karagiannidis et al. 2002). BCA Purpureocillium lilacinum QLP12 promoted seed germination $(76.7 \%)$, bud growth $(79.4 \%)$, chlorophyll content $(47.83 \%)$, and root activity $(182.02 \%)$, which altogether led to a significant decline in Verticillium wilt incidence by $83.82 \%$ in eggplant (Lan et al. 2017). Thus, the applied BCAs could have contributed to better nutrient uptake, healthier plants, better plant canopy, and reduced $V$. dahliae colonization. In addition, a strong correlation between biomass and plant height in both eggplant and tomato could be taken as an indicator of low Verticillium wilt incidence and severity and, thus, can be utilized to avoid labor-intensive disease scoring in the greenhouse or the field.

Several factors, including ecological fitness, host resistance, pathogen inoculum load, and so on, might play a role in a BCA's success (Deketelaere et al. 2017). T. flavus showed better protection when coinoculated in eggplant because it is capable of producing glucose oxidase, which has a lethal effect on the microsclerotia (Kim et al. 1988; Stosz et al. 1996) and inhibits microsclerotial germination (Marois et al.
1982). The lower and similar efficacy in tomato and nonsignificant interaction to inoculation time points in lettuce against VdLs16 signifies a host-dependent interaction of $T$. flavus. Integrating enhanced GFP in biocontrol and Discosoma Red Fluorescent Protein reporter genes in pathogenic strains, Pantelides et al. (2009) concluded that $F$. oxysporum strain F2 suppressed $V$. dahliae via increasing competition for space or nutrients on the root surface as the primary mode of action. It is quite likely that competition for space and nutrients was the primary mode of action observed in this study for $V$. isaacii and $V$. klebahnii rather than antagonism, as in T. flavus.

Many plant vascular system colonizing endophytes also induce PR proteins that activate systemic acquired resistance or induced systemic resistance in Verticillium interactions in hosts such as tomato, eggplants, and Arabidopsis (Antonopoulos et al. 2008; Tjamos et al. 2005; van Loon et al. 2006). For example, pepper plants inoculated with $V$. dahliae following inoculations with the nonpathogenic $F$. oxysporum (Fo47) showed a higher relative expression of PR-1 protein (CABPR1), a class II chitinase (CACHI2), and a sesquiterpene cyclase in root and stem tissues (Veloso and Díaz 2012). The soil application of 5\% dry mycelium of Penicillium chrysogenum provided resistance to $V$. dahliae in cotton by inducing peroxidase and lignin deposition in hypocotyls (Dong et al. 2003). The Paenibacillus alvei K165 and the nonpathogenic $F$. oxysporum $\mathrm{F} 2$ enhanced the expression of PR1 and PR4 in eggplant as a $V$. dahliae defense response (Angelopoulou et al. 2014). In contrast, our results showed an opposite association, where the application of BCAs decreased the level of PR protein-related gene expression in pathogen-challenged treatments. Such upregulation of defense-related genes agrees with a host-susceptibility model or constitutive resistance rather than induced resistance (Mandelc et al. 2013). The host plants without BCA were easily infected by $V$. dahliae and quickly mounted a robust defensive response via upregulation of induced resistance genes at the early infection stage (until $48 \mathrm{~h}$ ) but, ultimately, failed as the pathogen was established. Such noticeable changes in protein levels or expression of other known plant defense proteins, including PR-5, PR-10, chitinase, and $\beta$-glucanase in cotton and PR-2, PR-4, and so on in oilseed rape, were induced significantly in susceptible plants but only a modest variation was seen in a resistant plant in response to $V$. dahliae (McFadden et al. 2001; Robb et al. 2012; Xu et al. 2011). A systemic increase in plant defense proteins PR-1, chitinase (PR-3), $\beta-1,3$-glucanase (PR-2), and a thaumatin-like protein (PR-5) was significant in susceptible hop plants in response to a highly virulent $V$. albo-atrum strain (Cregeen et al. 2015). The PR-1 protein upregulated 153 -fold in susceptible but 1.5 -fold in resistant hops plants at 10 dpi (Cregeen et al. 2015). Robb et al. (2007) found a similar phenomenon, where genes strongly expressed in susceptible interactions were downregulated in tolerant tomato in response to $V$. dahliae. Klosterman et al. (2011) reported a similar relationship, where $P R-3, P R-5$, and a cysteine protease $(L s C P 2)$ were expressed only in symptomatic lettuce leaves at 3 wpi with $V$. dahliae. The PR protein genes Chi3, Chi9, GluA, GluB, and PR-1a had significantly low expression in the plants or cells inoculated with biocontrol strain $F$. oxysporum Fo47 (Aimé et al. 2008). Thus, it is likely that the biocontrol strains excluded $V$. dahliae from the root surface (Shittu et al. 2009; Tyvaert et al. 2014), preventing pathogen recognition. This assumption was strongly supported by our tracking of the GFP-tagged pathogen, where only limited colonization was observed in both eggplant and tomato vascular 
systems in the presence of BCAs. Despite these studies conducted on the most susceptible cultivars of each host, the BCAs reduced the disease caused by the most virulent strains. Combining BCAs with even moderately resistant cultivars of individual crops may dramatically reduce the incidence of Verticillium wilt on these crops. Thus, priming tomato plants with BCA PD659 could reduce disease establishment and provide cross-protection by limiting the pathogen's niche resources.

In conclusion, we evaluated the biocontrol efficacy of $V$. isaacii isolate PD660 and V. klebahnii isolate PD659, two species that are known to be endophytes and are occasionally isolated from asymptomatic lettuce or spinach plants (Gurung et al. 2015). Both BCAs resulted in significant control of the highly virulent isolate VdLs16 or other host-dependent isolates derived from the three crops evaluated in this study. Of the treatments evaluated, V. klebahnii isolate PD659, when introduced 2 weeks before $V$. dahliae inoculation, showed a significantly high biological control effect, compared with $T$. flavus and $V$. isaacii. Competitive exclusion might be the primary mode of action where disease initiation was delayed in a host-dependent manner. In light of these findings, seed coating of dicotyledenous crops with $V$. klebahnii isolate PD659 or soil-plug inoculation at least 2 weeks before transplanting could provide an alternative management tool against Verticillium wilt, which will eventually be a part of an integrated Verticillium wilt management. However, further research and optimization tests will be needed to develop these BCAs as a seed coating. We recognize that genetic exchanges between pathogenic and biocontrol Verticillium spp. strains have not been evaluated yet and, thus, should be taken under consideration before a large-scale introduction of these novel nonpathogenic strains on a commercial scale.

\section{ACKNOWLEDGMENTS}

We thank R. Marchebout for assistance with the greenhouse experiments and N. Carrasquilla-Garcia for her confocal microscopy expertise.

\section{LITERATURE CITED}

Aimé, S., Cordier, C., Alabouvette, C., and Olivain, C. 2008. Comparative analysis of PR gene expression in tomato inoculated with virulent Fusarium oxysporum f. sp. lycopersici and the biocontrol strain F. oxysporum Fo47. Physiol. Mol. Plant Pathol. 73:9-15.

Alexander, L. J. 1962. Susceptibility of certain Verticillium resistant tomato varieties to an Ohio isolate of the fungus. Phytopathology 52:998-1000

Angelopoulou, D. J., Naska, E. J., Paplomatas, E. J., and Tjamos, S. E. 2014. Biological control agents (BCAs) of Verticillium wilt: Influence of application rates and delivery method on plant protection, triggering of host defence mechanisms and rhizosphere populations of BCAs. Plant Pathol. 63:1062-1069.

Antonopoulos, D. F., Tjamos, S. E., Antoniou, P. P., Rafeletos, P., and Tjamos, E. C. 2008. Effect of Paenibacillus alvei, strain K165, on the germination of Verticillium dahliae microsclerotia in planta. Biol. Control 46:166-170.

Atallah, Z. K., Hayes, R. J., and Subbarao, K. V. 2011. Fifteen years of Verticillium wilt of lettuce in America's salad bowl: A tale of immigration, subjugation, and abatement. Plant Dis. 95:784-792.

Balaji, V., Mayrose, M., Sherf, O., Jacob-Hirsch, J., Eichenlaub, R., Iraki, N., Manulis-Sasson, S., Rechavi, G., Barash, I., and Sessa, G. 2008. Tomato transcriptional changes in response to Clavibacter michiganensis subsp. michiganensis reveal a role for ethylene in disease development. Plant Physiol. 146:1797-1809.

Bale, J. S., van Lenteren, J. C., and Bigler, F. 2008. Biological control and sustainable food production. Philos. Trans. R. Soc. Lond. B 363:761-776.

Barda, O., Shalev, O., Alster, S., Buxdorf, K., Gafni, A., and Levy, M. 2015. Pseudozyma aphidis induces salicylic-acid-independent resistance to Clavibacter michiganensis in tomato plants. Plant Dis. 99:621-626.

Berlanger, I., and Powelson, M. L. 2000. Verticillium wilt. Plant Health Instr. https://www.apsnet.org/edcenter/disandpath/fungalasco/pdlessons/ Pages/VerticilliumWilt.aspx

Block, A., Schmelz, E., O’Donnell, P. J., Jones, J. B., and Klee, H. J. 2005. Systemic acquired tolerance to virulent bacterial pathogens in tomato. Plant Physiol. 138:1481-1490.

Brunner, E., and Langer, F. 2000. Nonparametric analysis of ordered categorical data in designs with longitudinal observations and small sample sizes. Biom. J. 42:663-675.

Chellemi, D. O., Gamliel, A., Katan, J., and Subbarao, K. V. 2016. Development and deployment of systems-based approaches for the management of soil-borne plant pathogens. Phytopathology 106:216-225.

Chen, P., Lee, B., and Robb, J. 2004. Tolerance to a non-host isolate of Verticillium dahliae in tomato. Physiol. Mol. Plant Pathol. 64:283-291.

Cregeen, S., Radisek, S., Mandelc, S., Turk, B., Stajner, N., Jakse, J., and Javornik, B. 2015. Different gene expressions of resistant and susceptible hop cultivars in response to infection with a highly aggressive strain of Verticillium albo-atrum. Plant Mol. Biol. Rep. 33:689-704.

Davis, J., and Sorensen, L. 1985. Association of Verticillium tricorpus with Verticillium dahliae and potato early dying. (Abstr.) Phytopathology 75:1279.

Davis, J. R., Everson, D. O., Sorensen, L. H., and Schneider, A. T. 2000 Associations of Verticillium tricorpus with soil suppressiveness of Verticillium wilt of potato. Pages 347-351 in: Advances in Verticillium Research and Disease Management. E. C. Tjamos, R. C. Rowe, J. B. Heale, and D. R. Fravel, eds. American Phytopathological Society, St. Paul, MN, U.S.A.

Deketelaere, S., Spiessens, K., Pollet, S., Tyvaert, L., Rooster, L. D., Callens, D., França, S. C., and Höfte, M. 2020. Towards practical application of Verticillium isaacii vt305 to control Verticillium wilt of cauliflower: Exploring complementary biocontrol strategies. Plants 9:1469.

Deketelaere, S., Tyvaert, L., França, S. C., and Höfte, M. 2017. Desirable traits of a good biocontrol agent against Verticillium wilt. Front. Microbiol. 8:1186.

Demir, S., Şensoy, S., Ocak, E., Tüfenkci, S.., Durak, E. D., Erdinc, C., and Ünsal, H. 2015. Effects of arbuscular mycorrhizal fungus, humic acid, and whey on wilt disease caused by Verticillium dahliae Kleb. in three solanaceous crops. Turk. J. Agric. For. 39:300-309.

Dong, H., Li, W., Zhang, D., and Tang, W. 2003. Differential expression of induced resistance by an aqueous extract of killed Penicillium chrysogenum against Verticillium wilt of cotton. Crop Prot. 22:129-134.

Donley, N. 2019. The USA lags behind other agricultural nations in banning harmful pesticides. Environ. Health 18:44.

Duniway, J. 2002. Status of chemical alternatives to methyl bromide for pre-plant fumigation of soil. Phytopathology 92:1337-1343.

El-Hassan, S. A., and Gowen, S. R. 2006. Formulation and delivery of the bacterial antagonist Bacillus subtilis for management of lentil vascular wilt caused by Fusarium oxysporum f. sp. lentis. J. Phytopathol. 154:148-155.

Eljounaidi, K., Lee, S. K., and Bae, H. 2016. Bacterial endophytes as potential biocontrol agents of vascular wilt diseases- review and future prospects. Biol. Control 103:62-68.

França, S. C., Spiessens, K., Pollet, S., Debode, J., De Rooster, L., Callens, D., and Höfte, M. 2013. Population dynamics of Verticillium species in cauliflower fields: Influence of crop rotation, debris removal and ryegrass incorporation. Crop Prot. 54:134-141.

Fravel, D. R. 1989. Biocontrol of Verticillium wilt of eggplant and tomato. Pages 487-492 in: Vascular Wilt Diseases of Plants. E. C. Tjamos and C. H. Beckman, eds. Nato ASI Series, Vol. H28. Springer Verlag, Berlin, Germany.

Fravel, D. R. 2005. Commercialization and implementation of biocontrol. Annu. Rev. Phytopathol. 43:337-359. 
Gkizi, D., Lehmann, S., L'Haridon, F., Serrano, M., Paplomatas, E. J., Métraux, J. P., and Tjamos, S. E. 2016. The innate immune signaling system as a regulator of disease resistance and induced systemic resistance activity against Verticillium dahliae. Mol. Plant-Microbe Interact. 29:313-323.

Gurung, S., Short, D. P. G., Hu, X., Sandoya, G. V., Hayes, R. J., Koike, S. T., and Subbarao, K. V. 2015. Host range of Verticillium isaacii and Verticillium klebahnii from artichoke, spinach, and lettuce. Plant Dis. 99:933-938.

Han, Q., Wu, F., Wang, X., Qi, H., Shi, L., Ren, A., Liu, Q., Zhao, M., and Tang, C. 2015. The bacterial lipopeptide iturins induce Verticillium dahliae cell death by affecting fungal signalling pathways and mediate plant defence responses involved in pathogen-associated molecular pattern-triggered immunity. Environ. Microbiol. 17:1166-1188.

Hayes, R. J., Maruthachalam, K., Vallad, G. E., Klosterman, S. J., and Subbarao, K. V. 2011a. Selection for resistance to Verticillium wilt caused by race 2 isolates of Verticillium dahliae in accessions of lettuce (Lactuca sativa L.). HortScience 46:201-206.

Hayes, R. J., McHale, L. K., Vallad, G. E., Truco, M. J., Michelmore, R. W., Klosterman, S. J., Maruthachalam, K., and Subbarao, K. V. 2011b. The inheritance of resistance to Verticillium wilt caused by race 1 isolates of Verticillium dahliae in the lettuce cultivar La Brillante. Theor. Appl. Genet. 123:509-517.

Hayes, R. J., Vallad, G. E., Qin, Q. M., Grube, R. C., and Subbarao, K. V. 2007. Variation for resistance to Verticillium wilt in lettuce (Lactuca sativa L.). Plant Dis. 91:439-445.

Herman, M. A., Davidson, J. K., and Smart, C. D. 2008. Induction of plant defense gene expression by plant activators and Pseudomonas syringae pv. tomato in greenhouse-grown tomatoes. Phytopathology 98:1226-1232.

Inderbitzin, P., Bostock, R. M., Davis, R. M., Usami, T., Platt, H. W., and Subbarao, K. V. 2011. Phylogenetics and taxonomy of the fungal vascular wilt pathogen Verticillium, with the descriptions of five new species. PLoS One 6:e28341.

Karagiannidis, N., Bletsos, F., and Stavropoulos, N. 2002. Effect of Verticillium wilt (Verticillium dahliae Kleb.) and mycorrhiza (Glomus mosseae) on root colonization, growth and nutrient uptake in tomato and eggplant seedlings. Sci. Hortic. (Amsterdam) 94:145-156.

Keinath, A., Fravel, D., and Papavizas, G. 1991. Potential of Gliocladium roseum for biocontrol of Verticillium dahliae. Phytopathology 81: 644-648.

Kim, K., Fravel, D., and Papavizas, G. 1988. Identification of a metabolite produced by Talaromyces flavus as glucose oxidase and its role in the biocontrol of Verticillium dahliae. Phytopathology 78:488-492.

Klosterman, S. J., Anchieta, A., Garcia-Pedrajas, M. D., Maruthachalam, K., Hayes, R. J., and Subbarao, K. V. 2011. SSH reveals a linkage between a senescence-associated protease and Verticillium wilt symptom development in lettuce (Lactuca sativa). Physiol. Mol. Plant Pathol. 76:48-58.

Klosterman, S. J., Atallah, Z. K., Vallad, G. E., and Subbarao, K. V. 2009. Diversity, pathogenicity, and management of Verticillium species. Annu. Rev. Phytopathol. 47:39-62.

Lan, X., Zhang, J., Zong, Z., Ma, Q., and Wang, Y. 2017. Evaluation of the biocontrol potential of Purpureocillium lilacinum QLP12 against Verticillium dahliae in eggplant. BioMed Res. Int. 2017:4101357.

Li, S., Zhang, N., Zhang, Z., Luo, J., Shen, B., Zhang, R., and Shen, Q. 2013. Antagonist Bacillus subtilis HJ5 controls Verticillium wilt of cotton by root colonization and biofilm formation. Biol. Fertil. Soils 49:295-303.

Madi, L., Katan, T., Katan, J., and Henis, Y. 1997. Biological control of Sclerotium rolfsii and Verticillium dahliae by Talaromyces flavus is mediated by different mechanisms. Phytopathology 87:1054-1060.

Malandraki, I., Tjamos, S. E., Pantelides, I. S., and Paplomatas, E. J. 2008. Thermal inactivation of compost suppressiveness implicates possible biological factors in disease management. Biol. Control 44:180-187.

Malcolm, G. M., Kuldau, G. A., Gugino, B. K., and Jiménez-Gasco Mdel, M. 2013. Hidden host plant associations of soil-borne fungal pathogens: An ecological perspective. Phytopathology 103:538-544.

Mandelc, S., Timperman, I., Radišek, S., Devreese, B., Samyn, B., and Javornik, B. 2013. Comparative proteomic profiling in compatible and incompatible interactions between hop roots and Verticillium alboatrum. Plant Physiol. Biochem. 68:23-31.

Marois, J. J., Fravel, D. R., and Papavizas, G. C. 1984. Ability of Talaromyces flavus to occupy the rhizosphere and its interaction with Verticillium dahliae. Soil Biol. Biochem. 16:387-390.

Marois, J. J., Johnston, S. A., Dunn, M. T., and Papavizas, G. C. 1982. Biological control of Verticillium wilt of eggplant in the field. Plant Dis. 66:1166-1168.

Martin, F. N. 2003. Development of alternative strategies for management of soil-borne pathogens currently controlled with methyl bromide. Annu. Rev. Phytopathol. 41:325-350.

Maruthachalam, K., Klosterman, S. J., Anchieta, A., Mou, B., and Subbarao, K. V. 2013. Colonization of spinach by Verticillium dahliae and effects of pathogen localization on the efficacy of seed treatments. Phytopathology 103:268-280.

McFadden, H. G., Chapple, R., de Feyter, R., and Dennis, E. 2001. Expression of pathogenesis-related genes in cotton stems in response to infection by Verticillium dahliae. Physiol. Mol. Plant Pathol. 58:119-131.

Meadows, R. 2013. News overview: Researchers develop alternatives to methyl bromide fumigation. Calif. Agric. 67:125-127.

Müller, H., and Berg, G. 2008. Impact of formulation procedures on the effect of the biocontrol agent Serratia plymuthica HRO-C48 on Verticillium wilt in oilseed rape. BioControl 53:905-916.

Nagtzaam, M. P. M., Bollen, G. J., and Termorshuizen, A. J. 1998. Efficacy of Talaromyces flavus alone or in combination with other antagonists in controlling Verticillium dahliae in growth chamber experiments. J. Phytopathol. 146:165-173.

Naraghi, L., Heydari, A., Rezaee, S., Razavi, M., Jahanifar, H., and Khaledi, E. 2010. Biological control of tomato Verticillium wilt disease by Talaromyces flavus. J. Plant Prot. Res. 50:360-365.

Pantelides, I. S., Tjamos, S. E., Striglis, I. A., Chatzipavlidis, I., and Paplomatas, E. J. 2009. Mode of action of a non-pathogenic Fusarium oxysporum strain against Verticillium dahliae using Real Time QPCR analysis and biomarker transformation. Biol. Control 50:30-36.

Pegg, G. F., and Brady, B. L., eds. 2002. Verticillium Wilts. CABI Publishing, Wallingford, United Kingdom.

Pfaffl, M. W., Horgan, G. W., and Dempfle, L. 2002. Relative expression software tool (RESTC) for group-wise comparison and statistical analysis of relative expression results in real-time PCR. Nucleic Acids Res. 30:e36.

Postma, J., and Goossen-van de Geijn, H. 2016. Twenty-four years of Dutch Trig® application to control Dutch elm disease. BioControl 61:305-312.

Puri, K. D., Gurung, S., Short, D. P. G., Atallah, Z., Sandoya, G., Davis, M., Hayes, R. J., and Subbarao, K. V. 2017. Short-term host selection pressure has little effect on the evolution of a monoclonal population of Verticillium dahliae race 1. Phytopathology 107:1417-1425.

Puri, K. D., Yan, C., Leng, Y., and Zhong, S. 2016. RNA-Seq revealed differences in transcriptomes between 3ADON and 15ADON populations of Fusarium graminearum in vitro and in planta. PLoS One 11:e0163803.

Qin, Q. M., Vallad, G. E., and Subbarao, K. V. 2008. Characterization of Verticillium dahliae and $V$. tricorpus isolates from lettuce and artichoke. Plant Dis. 92:69-77.

Robb, J., Lee, B., and Nazar, R. N. 2007. Gene suppression in a tolerant tomato-vascular pathogen interaction. Planta 226:299-309.

Robb, J., Shittu, H., Soman, K. V., Kurosky, A., and Nazar, R. N. 2012. Arsenal of elevated defense proteins fails to protect tomato against Verticillium dahliae. Planta 236:623-633.

Robinson, N., Piatt, H. W., and Hale, L. R. 2007. Verticillium dahliae interactions with $V$. albo-atrum and $V$. tricorpus and their effects on Verticillium wilt disease development in potato. Am. J. Potato Res. 84:229-235.

Rocha, I., Ma, Y., Souza-Alonso, P., Vosátka, M., Freitas, H., and Oliveira, R. S. 2019. Seed coating: A tool for delivering beneficial microbes to agricultural crops. Front. Plant Sci. 10:1357.

Sandoya, G., Truco, M. J., Bertier, L. D., Subbarao, K. V., Simko, I., Hayes, R. J., and Michelmore, R. Genetics of partial resistance against Verticillium dahliae race 2 in wild and cultivated lettuce. Phytopathology. In press. 
Shah, D. A., and Madden, L. V. 2004. Nonparametric analysis of ordinal data in designed factorial experiments. Phytopathology 94:33-43.

Shittu, H. O., Castroverde, D. C. M., Nazar, R. N., and Robb, J. 2009. Plant-endophyte interplay protects tomato against a virulent Verticillium. Planta 229:415-426.

Short, D. P., Gurung, S., Koike, S. T., Klosterman, S. J., and Subbarao, K. V. 2015. Frequency of Verticillium species in commercial spinach fields and transmission of $V$. dahliae from spinach to subsequent lettuce crops. Phytopathology 105:80-90.

Stosz, S. K., Fravel, D. R., and Roberts, D. P. 1996. In vitro analysis of the role of glucose oxidase from Talaromyces flavus in biocontrol of the plant pathogen Verticillium dahliae. Appl. Environ. Microbiol. 62:3183-3186.

Subbarao, K. V., Davis, R. M., Gilbertson, R. L., and Raid, R. N. 2017. Compendium of Lettuce Diseases and Pests, 2nd Ed. American Phytopathological Society, St. Paul, MN, U.S.A.

Taylor, J. W., Jacobson, D. J., Kroken, S., Kasuga, T., Geiser, D. M., Hibbett, D. S., and Fisher, M. C. 2000. Phylogenetic species recognition and species concepts in fungi. Fungal Genet. Biol. 31:21-32.

Tjamos, E. C., and Fravel, D. R. 1995. Detrimental effects of sublethal heating and Talaromyces flavus on microsclerotia of Verticillium dahliae. Phytopathology 85:388-392.

Tjamos, S. E., Flemetakis, E., Paplomatas, E. J., and Katinakis, P. 2005. Induction of resistance to Verticillium dahliae in Arabidopsis thaliana by the biocontrol agent K-165 and pathogenesis-related proteins gene expression. Mol. Plant-Microbe Interact. 18:555-561.

Tyvaert, L., França, S. C., Debode, J., and Höfte, M. 2014. The endophyte Verticillium Vt305 protects cauliflower against Verticillium wilt. J. Appl. Microbiol. 116:1563-1571.

Usami, T., Kanto, T., Inderbitzin, P., Itoh, M., Kisaki, G., Ebihara, Y., Suda, W., Amemiya, Y., and Subbarao, K. V. 2011. Verticillium tricorpus causing lettuce wilt in Japan differs genetically from California lettuce isolates. J. Gen. Plant Pathol. 77:17-23.
Usami, T., Momma, N., Kikuchi, S., Watanabe, H., Hayashi, A., Mizukawa, M., Yoshino, K., and Ohmori, Y. 2017. Race 2 of Verticillium dahliae infecting tomato in Japan can be split into two races with differential pathogenicity on resistant rootstocks. Plant Pathol. 66:230-238.

Vallad, G. E., and Subbarao, K. V. 2008. Colonization of resistant and susceptible lettuce cultivars by a green fluorescent protein-tagged isolate of Verticillium dahliae. Phytopathology 98:871-885.

van Loon, L. C., Rep, M., and Pieterse, C. M. J. 2006. Significance of inducible defense-related proteins in infected plants. Annu. Rev. Phytopathol. 44:135-162.

Veloso, J., and Díaz, J. 2012. Fusarium oxysporum Fo47 confers protection to pepper plants against Verticillium dahliae and Phytophthora capsici, and induces the expression of defence genes. Plant Pathol. 61:281-288.

Xu, L., Zhu, L., Tu, L., Guo, X., Long, L., Sun, L., Gao, W., and Zhang, X. 2011. Differential gene expression in cotton defence response to Verticillium dahliae by SSH. J. Phytopathol. 159:606-615.

Yuan, Y., Feng, H., Wang, L., Li, Z., Shi, Y., Zhao, L., Feng, Z., and Zhu, H. 2017. Potential of endophytic fungi isolated from cotton roots for biological control against Verticillium wilt disease. PLoS One 12: 0170557

Zare, R., Gams, W., Starink-Willemse, M., and Summerbell, R. 2007. Gibellulopsis, a suitable genus for Verticillium nigrescens, and Musicillium, a new genus for $V$. theobromae. Nova Hedwigia 85:463-489.

Zeise, K., and Kersten, H. 2000. The potential of Talaromyces flavus in controlling Verticillium dahliae. Pages 232-236 in: Advances in Verticillium Research and Disease Management. E. C. Tjamos, R. C. Rowe, J. B. Heale, and D. R. Fravel, eds. American Phytopathological Society, St. Paul, MN, U.S.A.

Zhu, H. Q., Feng, Z. L., Li, Z. F., Shi, Y. Q., Zhao, L. H., and Yang, J. R. 2013. Characterization of two fungal isolates from cotton and evaluation of their potential for biocontrol of Verticillium wilt of cotton. J. Phytopathol. 161:70-77. 